\title{
Randomized Algorithms for Probabilistic Robustness with Real and Complex Structured Uncertainty
}

\author{
Giuseppe C. Calafiore, Fabrizio Dabbene, and Roberto Tempo, Fellow, IEEE
}

\begin{abstract}
In recent years, there has been a growing interest in developing randomized algorithms for probabilistic robustness of uncertain control systems. Unlike classical worst case methods, these algorithms provide probabilistic estimates assessing, for instance, if a certain design specification is met with a given probability. One of the advantages of this approach is that the robustness margins can be often increased by a considerable amount, at the expense of a small risk. In this sense, randomized algorithms may be used by the control engineer together with standard worst case methods to obtain additional useful information.

The applicability of these probabilistic methods to robust control is presently limited by the fact that the sample generation is feasible only in very special cases which include systems affected by real parametric uncertainty bounded in rectangles or spheres. Sampling in more general uncertainty sets is generally performed through overbounding, at the expense of an exponential rejection rate.

In this paper, randomized algorithms for stability and performance of linear time invariant uncertain systems described by a general $M-\Delta$ configuration are studied. In particular, efficient polynomial-time algorithms for uncertainty structures $\Delta$ consisting of an arbitrary number of full complex blocks and uncertain parameters are developed.
\end{abstract}

Index Terms-Random matrices, randomized algorithms, robust control, uncertainty.

\section{INTRODUCTION}

$\mathbf{T}$ HE most frequently used configuration for robustness analysis and design of complex uncertain feedback systems is the so-called $M-\Delta$ model; see, e.g., [42]. This model consists of a given plant $M$, which possibly includes weighting functions and a controller, and an uncertain block diagonal matrix $\Delta$. With this feedback configuration, different design methodologies can be implemented and several performance objectives can be analyzed. In addition, since the structure of $\Delta$ is very general, various sources of uncertainty, such as parametric, nonparametric, structured and unstructured, can be easily taken into account. For these reasons, the $M-\Delta$ configuration is a valuable tool for both practitioners and theoreticians, so that powerful and useful results have been developed in the last few years. At the same time, however, classical worst case robust control has also shown some limitations when the control system is affected by general uncertainty structures $\Delta$. To investigate these limitations, many papers focused on

Manuscript received February 11, 1999; revised November 20, 1999. Recommended by Associate Editor, X. Zhou. This work was supported in part by IRITI-CNR, under Grant CNR 99.01960.CT07 and by funds of MURST.

The authors are with the Dipartimento di Automatica e Informatica, IRITI-CNR, Politecnico di Torino, 10129 Torino, Italy (e-mail: calafiore@polito.it; dabbene@polito.it; tempo@polito.it).

Publisher Item Identifier S 0018-9286(00)10666-X. complexity issues of feedback system; see [5], [7], [15], [28], and [30]. The contribution of these papers is to demonstrate that several problems in linear robust control are NP-hard, which in turn implies that they are not practically tractable, unless the number of uncertainties entering into the feedback system is very limited. To avoid this drawback, many other contributions attacked the same problem following a parallel line of research, with the goal of computing upper and lower bounds (instead of the "true" value) of the robustness margin for very general feedback configurations. In other words, the focal point of these papers is to develop either necessary or sufficient conditions for robust stability and performance. The nice feature of these bounds is that their evaluation generally requires the solution of convex programs which can be easily performed, for example, by means of interior point methods [6]. However, the issue of conservatism is still present.

In order to overcome these difficulties, a different paradigm has recently emerged. This new paradigm studies uncertain feedback systems from a probabilistic point of view; see, e.g., [3], [13], [25], [31], [41], and [43]; additional references can be found in the survey papers [38] and [39]. This framework is not alternative to worst case robust control, but it provides useful and complementary information to the control engineer. In this setting, $\Delta$ is indeed bounded in a given set, but it is also a random matrix with a given probability distribution. In this way, both probabilistic and deterministic information is captured.

In this paper, the class of so-called radially symmetric distributions over the uncertainty set is studied. Roughly speaking, a radially symmetric distribution has the property that the density function $f_{\Delta}$ of $\Delta$ depends only on the spectral norm of $\Delta$; see Section III and [4] for a rigorous definition, and the papers [2] and [3] for further guidelines on the choice of the distribution. This class includes as a special case the uniform distribution in a bounded support set defined as in classical worst case robust control. That is, we consider the set of matrices $\Delta$ whose spectral norm is bounded by $\rho>0$

$$
\{\Delta: \bar{\sigma}(\Delta) \leq \rho\}
$$

where $\bar{\sigma}(\Delta) \doteq \max _{i} \sigma_{i}(\Delta)=\|\Delta\|_{\sigma}$. In addition, the matrix $\Delta$ is structured so that it contains real and complex parameters and real and complex full blocks; see Section II for a precise definition.

Within this framework, the main task of this paper is to develop randomized algorithms for stability and performance analysis of linear time invariant uncertain systems. To this end, we consider a state space realization $(A ; B ; C ; D)$ of $M$, 
and study the stability of $A+B \Delta(I-D \Delta)^{-1} C$. Frequency domain and state space interpretations of such stability tests have been provided in the $M-\Delta$ setting see, e.g., [32] and [42]. For example, if $M(s)=C(s I-A)^{-1} B+D, A$ is stable and the well-posedness condition on $D$ holds, then

$$
\begin{aligned}
& \left\{\Delta: A+B \Delta(I-D \Delta)^{-1} C \text { is stable }\right\} \\
& \quad=\{\Delta: \operatorname{det}(I-\Delta M(j \omega)) \neq 0, \forall \omega \in \mathbb{R}\} .
\end{aligned}
$$

We remark that, when $\Delta$ consists of a single block, the size of the smallest destabilizing perturbation is often called the stability radius [21], [32]. In this sense, this paper studies the probabilistic version of the stability radius problem for the case when the system is affected by general uncertainty structures $\Delta$. A parallel line of research, not directly related to the problem formulation and results given in this paper, is focused on the computation of the stability radius of systems subject to stochastic perturbations; see [19].

Formally, for fixed $\rho>0$, we let

$$
p(\rho) \doteq \operatorname{Prob}\left\{A+B \Delta(I-D \Delta)^{-1} C \text { is stable }\right\}
$$

and, for given $p^{*} \in[0,1]$, we define the probabilistic stability margin

$$
\rho\left(p^{*}\right) \doteq \sup \left\{\rho: p(\rho) \geq p^{*}\right\} .
$$

Given a probability level $p^{*}$, the probabilistic stability margin $\rho\left(p^{*}\right)$ gives the maximum size of the perturbation $\Delta$, measured according to the spectral norm, so that the probability $p(\rho)$ is at least $p^{*}$. Once $p(\rho)$ is computed, the next step is to construct the probability degradation function, i.e., the plot of the probability of stability as a function of the radius $\rho$. This plot may be compared with the classical worst case stability margin $1 / \mu$, obtaining

$$
\rho\left(p^{*}\right) \geq 1 / \mu
$$

for any $p^{*} \in[0,1]$. This fact in turn implies that the margin computed with probabilistic methods is always larger than the classical worst case margin, at the expense of a risk expressed in probability.

In order to compute $p(\rho)$ with randomized algorithms, we generate $N$ matrix samples

$$
\Delta^{1}, \Delta^{2}, \ldots, \Delta^{N}
$$

in the set $\{\Delta: \bar{\sigma}(\Delta) \leq \rho\}$ according to $f_{\Delta}(\Delta)$ and the structure of $\Delta$. Then, we construct an indicator function

$$
I\left(\Delta^{i}\right)= \begin{cases}1, & \text { if } A+B \Delta^{i}\left(I-D \Delta^{i}\right)^{-1} C \text { is stable } \\ 0, & \text { otherwise }\end{cases}
$$

and compute the empirical probability

$$
\hat{p}_{N}(\rho)=\frac{1}{N} \sum_{i=1}^{N} I\left(\Delta^{i}\right) .
$$

We recall that the Chernoff bound [14] gives the sample size $N$ so that the empirical probability $\hat{p}_{N}(\rho)$ is "close" to $p(\rho)$ with accuracy $\epsilon$ and confidence $\delta$. Formally, for any $\epsilon \in(0,1)$ and $\delta \in(0,1)$, if

$$
N \geq \frac{\log \frac{2}{\delta}}{2 \epsilon^{2}}
$$

then

$$
\operatorname{Prob}\left\{\left|p(\rho)-\hat{p}_{N}(\rho)\right| \leq \epsilon\right\} \geq 1-\delta .
$$

An obvious but crucial observation which follows immediately from the Chernoff bound is that $N$ is independent of the number and dimension of the uncertain blocks in the $\Delta$ matrix and of the size of the bounding set [24], [37]; similar statements can be also made when $\Delta$ consists of parametric uncertainty only [33]. The same conclusion holds when dealing with more sophisticated bounds arising in Learning Theory; see [40]. In either case, since checking the stability of $A+B \Delta^{i}\left(I-D \Delta^{i}\right)^{-1} C$ can be performed in polynomial-time, it follows that the overall complexity is also polynomial-time, provided that the cost of the generation of each matrix sample $\Delta^{i}$ is polynomial-time. With these motivations, the main objective of the paper is to efficiently generate matrix samples in the spectral norm ball according to a radially symmetric distribution and the structure of $\Delta$. We remark that the methods developed here are fundamentally different from the asymptotic techniques available for example in [26] for general convex sets, which are of limited use, since the number of samples given by the Chernoff bound is in general relatively small.

We now summarize the main results of the paper. In Section II, we introduce definitions and notation and we also show that the problem of generating samples of the structured matrix $\Delta$ is equivalent to sample generation in independent sets, each consisting of a full block matrix or a vector of parameters. Since the problem of the generation of random vectors has been already solved in [9] and [10], we study the case of a full block real or complex matrix $\Delta$. The starting point of this analysis is the singular value decomposition of $\Delta$. That is, we write $\Delta=U \Sigma V^{*}$, where $U$ and $V$ are unitary matrices and $\Sigma$ is a diagonal matrix containing the singular values of $\Delta$. Subsequently, for random matrices $\Delta$ with radially symmetric distribution, we compute the probability density functions (p.d.f.) of $f_{U}, f_{\Sigma}$, and $f_{V}$ of $U, \Sigma$, and $V$, respectively; see Theorems 1 and 2 in Section III. Basically, in these two theorems we prove that $f_{U}$ and $f_{V}$ are distributed according to the so-called Haar invariant distribution, while $f_{\Sigma}$ is a multivariate polynomial related to the determinant of a Vandermonde matrix. For the complex case, in Section IV we exploit the specific structure of this p.d.f. together with the well-known conditional method, in order to construct a polynomial-time algorithm for sample generation; see Algorithm 1. For the real case, the problem turns out to be more difficult and not easily tractable with these techniques and a rejection-based algorithm is presented in Section V. However, this algorithm uses the specific structure of $f_{\Sigma}$ and its rejection rate improves significantly upon that obtained by a straightforward set overbounding; see also [11]. In Section VI, we study a numerical example of a flexible structure with one complex block and two repeated real uncertain parameters and compute 
the probability degradation function using the proposed techniques. Concluding remarks are given in Section VII and the Appendix contains the proofs.

\section{DEFINITIONS AND NOTATION}

In this paper, we denote by $\Delta^{T}$ the transpose of $\Delta \in \mathbb{F}^{n, m}$, and by $\Delta^{*}$ its conjugate transpose, where $\mathbb{F}$ is either the complex field $\mathbb{C}$ or the real field $\mathbb{R}$. The notation $[\Delta]_{i, k}$ indicates the $(i, k)$ th element of $\Delta$ and, if $m=n,|\Delta|$ represents the absolute value of the determinant of $\Delta ; I_{n}$ denotes the $n \times n$ identity matrix, the subscript is omitted when the dimension can be deduced from the context. The spectral norm ball of radius $\rho>0$ is defined as

$$
\mathcal{B}_{\sigma}(\rho) \doteq\left\{\Delta \in \mathbb{F}^{n, m}: \bar{\sigma}(\Delta) \leq \rho\right\}
$$

Similarly, we denote the norm balls in the Frobenius and $\ell_{\infty}$ norms as $\mathcal{B}_{F}(\rho), \mathcal{B}_{\infty}(\rho)$.

The symbol $\Gamma(x)$ denotes the standard Gamma function and, for continuity, we let

$$
\left.\frac{\Gamma\left(\frac{x-1}{2}\right)}{\Gamma(x-1)}\right|_{x=1} \doteq 2
$$

Real and Complex Random Matrices: A real random matrix $\Delta \in \mathbb{R}^{n, m}$ is a matrix of random variables $[\Delta]_{i, k}$, $i=1, \ldots, n ; k=1, \ldots, m$. The probability density function $f_{\Delta}(\Delta)$ is defined as the joint probability density of the elements of $\Delta$. A complex random matrix $\Delta \in \mathbb{C}^{n, m}$ is defined as $\Delta=\operatorname{Re}(\Delta)+j \operatorname{Im}(\Delta)$, where $\operatorname{Re}(\Delta)$ and $\operatorname{Im}(\Delta)$ are real random matrices. The p.d.f. $f_{\Delta}(\Delta)$ is defined as the joint p.d.f. of $\operatorname{Re}(\Delta)$ and $\operatorname{Im}(\Delta)$.

The notation $\Delta \sim f_{\Delta}$ means that $\Delta$ is a random matrix with probability density $f_{\Delta} ; X \sim Y$ is also used to indicate that the two random matrices $X$ and $Y$ have the same p.d.f.

Uniform Density: For a measurable set $\mathcal{S}, \Delta \sim \mathcal{U}[\mathcal{S}]$ means that $\Delta$ is a random matrix with uniform density over the set $\mathcal{S}$. That is

$$
f_{\Delta}(\Delta)=\mathcal{U}[\mathcal{S}] \doteq \begin{cases}\frac{1}{\operatorname{vol}(S)}, & \text { if } \Delta \in \mathcal{S} \\ 0, & \text { otherwise }\end{cases}
$$

where $\operatorname{vol}(\mathcal{S})$ is the volume of the set $\mathcal{S}$. If $\mathcal{S}$ is in a linear space isomorphic to $\mathbb{R}^{d}$, we denote by volume the $d$-dimensional euclidean measure of $\mathcal{S}$.

Singular Value Decomposition (SVD): Given $\Delta \in \mathbb{F}^{n, m}$, $m \geq n$, we use the following normalized form of the SVD of $\Delta$

$$
\Delta=U \Sigma V^{*}
$$

where $U \in \mathbb{F}^{n, n}$ and $V \in \mathbb{F}^{m, n}$ have orthonormal columns, and $\Sigma=\operatorname{diag}\left(\sigma_{1}, \ldots, \sigma_{n}\right)$, with $\sigma_{1} \geq \sigma_{2} \geq \cdots \geq \sigma_{n} \geq 0$. The columns of $V$ are normalized so that the first nonvanishing component of each column is real and positive.
Radially Symmetric Densities: For $\Delta \in \mathbb{F}^{n, m}$, we consider the class of probability densities $f_{\Delta}$ that depend only on the largest singular value of $\Delta$

$$
\mathcal{F}_{\mathcal{R}} \doteq\left\{f_{\Delta}: f_{\Delta}(\Delta)=f_{\Delta}(\bar{\sigma}(\Delta))\right\}
$$

We remark that the uniform density in the spectral norm ball $\mathcal{B}_{\sigma}(\rho)$ of radius $\rho>0$ is in the class $\mathcal{F}_{\mathcal{R}}$, i.e.,

$$
\mathcal{U}\left[\mathcal{B}_{\sigma}(\rho)\right] \in \mathcal{F}_{\mathcal{R}}
$$

We now introduce another and more general class of density functions. This is the class $\mathcal{F}_{\mathcal{I}} \supset \mathcal{F}_{\mathcal{R}}$ of density functions which depend only on the singular values of $\Delta$. We call this class unitarily invariant. The name follows from the fact that if $\Delta \in \mathbb{C}^{n, m}$ has a unitarily invariant p.d.f., then $Q \Delta \sim \Delta$ and $\Delta W \sim \Delta$, for any given unitary matrices $Q$ and $W$.

Unitarily Invariant Densities: Let $\Delta \in \mathbb{F}^{n, m}$, the class of unitarily invariant p.d.f.s is defined as

$$
\mathcal{F}_{\mathcal{I}} \doteq\left\{f_{\Delta}(\Delta)=f_{\Delta}(\Sigma)\right\}
$$

\section{A. Structured Random Uncertainties}

As discussed in Section I, the matrix $\Delta$ is used to describe various perturbations affecting the control system. In robust control, the class of allowable perturbations is usually defined as in [42]

$$
\Delta \doteq\left\{\operatorname{blockdiag}\left[q_{1} I_{r_{1}}, \ldots, q_{s} I_{r_{s}}, \Delta_{1}, \ldots, \Delta_{b}\right]\right\}
$$

where $q_{i} \in \mathbb{F}, i=1, \ldots, s$ are scalar parameters with multiplicity $r_{1}, \ldots, r_{s}$, and $\Delta_{i} \in \mathbb{F}^{n_{i}, m_{i}}, i=1, \ldots, b$ are possibly repeated full blocks. The structured matrix $\Delta$ is restricted to the set

$$
\mathcal{B}_{\Delta}(\rho) \doteq\{\Delta \in \Delta: \bar{\sigma}(\Delta) \leq \rho\}
$$

that denotes the set of perturbations in $\Delta$ with size at most $\rho$. First, we notice that

$$
\bar{\sigma}(\Delta)=\max \left\{\|q\|_{\infty}, \bar{\sigma}\left(\Delta_{1}\right), \ldots, \bar{\sigma}\left(\Delta_{b}\right)\right\}
$$

where $q \doteq\left[q_{1}, \ldots, q_{s}\right]^{T}$ and $\|q\|_{\infty}=\max _{i}\left|q_{i}\right|$. Then, (8) implies that the vector $q$ is bounded in the set

$$
\left\{q:\|q\|_{\infty} \leq \rho\right\}
$$

and the full blocks $\Delta_{i}$ are restricted in

$$
\left\{\Delta_{i}: \bar{\sigma}\left(\Delta_{i}\right) \leq \rho\right\}, \quad i=1, \ldots, b .
$$

In this paper, we make the following assumptions.

Assumption 1: The vector $q$ and the blocks $\Delta_{i}$ are random vector and matrices with independent density functions.

Assumption 2: The p.d.f. of each matrix block $\Delta_{i} \in \mathbb{F}^{n_{i}, m_{i}}$ is unitarily invariant.

The independence assumption implies that the sampling problem in the ball $\mathcal{B}_{\Delta}(\rho)$ is equivalent to sampling independently the sets (9) and (10). For the vector case, sampling in the set (9) can be easily obtained with the techniques described in [9] and [10], for a class of probability densities having radial symmetry with respect to $\ell_{p}$ norms. The sampling problem 
TABLE I

REJECTION RATES FOR SQUARE COMPLEX MATRICES

\begin{tabular}{c|cccccccc}
\hline$n$ & 2 & 3 & 4 & 5 & 6 & 8 & 10 & 12 \\
\hline$\gamma_{\infty}$ & 12 & 8,640 & $870,912,000$ & $2 \mathrm{e} 16$ & $2 \mathrm{e} 26$ & $5 \mathrm{e} 54$ & $1 \mathrm{e} 95$ & $2 \mathrm{e} 148$ \\
\hline$\gamma_{F}$ & 8 & 468 & 178,780 & $4 \mathrm{e} 8$ & $6 \mathrm{e} 12$ & $2 \mathrm{e} 23$ & $1 \mathrm{e} 37$ & $1 \mathrm{e} 54$ \\
\hline
\end{tabular}

with several full blocks turns out to be much more difficult and is the main objective of this paper. Also in this case, however, the problem is reduced to sampling the set (10). For this reason, in the next sections we focus on the case of a single full matrix block $\Delta$. That is, for $\Delta \in \mathbb{F}^{n, m}$ we study sample generation in the set $\mathcal{B}_{\sigma}(\rho)$ according to $f_{\Delta}(\Delta)$.

To further motivate the results of this paper, suppose that one wants to generate uniform samples of square complex matrices in $\mathcal{B}_{\sigma}(1)$, using the classical method of rejection from an outer bounding set, where the sample generation is easier, as suggested in [24]. This is typically done considering for instance the following outer bounding sets

and

$$
\mathcal{B}_{F}(\sqrt{n})=\left\{\Delta \in \mathbb{C}^{n, n}:\|\Delta\|_{F} \leq \sqrt{n}\right\}
$$

$$
\mathcal{B}_{\infty}(1)=\left\{\Delta \in \mathbb{C}^{n, n}:\|\Delta\|_{\infty} \leq 1\right\}
$$

For these sets, it is well-known that $\mathcal{B}_{\sigma}(1) \subset \mathcal{B}_{F}(\sqrt{n})$ and $\mathcal{B}_{\sigma}(1) \subset \mathcal{B}_{\infty}(1)$; see [22]. Uniform sample generation in $\mathcal{B}_{F}$ and in $\mathcal{B}_{\infty}$ can be easily performed using the methods described in [10]. The samples that fall outside the set $\mathcal{B}_{\sigma}$ are then rejected. The rejection rate $\gamma$, which is the expected number of samples that should be generated in the outer set in order to find one sample in $\mathcal{B}_{\sigma}$, is simply given as the ratio of volumes

$$
\gamma_{F} \doteq \frac{\operatorname{vol}\left(\mathcal{B}_{F}(\sqrt{n})\right)}{\operatorname{vol}\left(\mathcal{B}_{\sigma}(1)\right)}, \quad \gamma_{\infty} \doteq \frac{\operatorname{vol}\left(\mathcal{B}_{\infty}(1)\right)}{\operatorname{vol}\left(\mathcal{B}_{\sigma}(1)\right)} .
$$

Table I shows that these rejection rates increase exponentially with the dimension $n$. The volume $\operatorname{vol}\left(\mathcal{B}_{\sigma}(1)\right)$ is computed according to the formula (22) derived in Section IV, while the formulas for the volumes of the Frobenius and infinity norm balls can be found for instance in [10].

Table I clearly shows the inefficiency of the rejection method, and motivates the need for more sophisticated techniques for direct generation of samples in $\mathcal{B}_{\sigma}(\rho)$.

\section{PROPERTIES OF UNITARILY INVARIANT DistribUtions}

In this section, we study the properties of the class of densities $\mathcal{F}_{\mathcal{I}}$, for full block complex and real matrices. In particular, we present two key results relating the probability density $f_{\Delta}(\Delta)$ with the probability density functions of the matrices $U, \Sigma$, and $V$ of the SVD of $\Delta$. We first consider the complex case.

Theorem 1: Let $\Delta \in \mathbb{C}^{n, m}, m \geq n \geq 2$, be factored as in (4), with $\sigma_{1}>\sigma_{2}>\cdots>\sigma_{n}>0$, and $[V]_{1, i}>0$, for $i=1, \ldots, n$. The following statements are equivalent.

1) The p.d.f. $f_{\Delta}(\Delta)$ is unitarily invariant, i.e., $f_{\Delta} \in \mathcal{F}_{\mathcal{I}}$.

2) The joint p.d.f. of $U, \Sigma$, and $V$ is

$$
f_{U, \Sigma, V}(U, \Sigma, V)=f_{U}(U) f_{\Sigma}(\Sigma) f_{V}(V)
$$

where

$$
\begin{gathered}
f_{U}(U)=\mathcal{U}\left[\left\{U: U^{*} U=I\right\}\right] \\
f_{\Sigma}(\Sigma)=\Upsilon_{\mathbb{C}} f_{\Delta}(\Sigma) \prod_{i=1}^{n} \sigma_{i}^{2(m-n)+1} \\
\prod_{1 \leq i<k \leq n}\left(\sigma_{i}^{2}-\sigma_{k}^{2}\right)^{2} \\
f_{V}(V)=\mathcal{U}\left[\left\{V: V^{*} V=I,[V]_{1, i}>0,\right.\right. \\
i=1, \ldots, n\}]
\end{gathered}
$$

and $\Upsilon_{\mathbb{C}}$ is a normalization constant given by

$$
\Upsilon_{\mathbb{C}}=\frac{2^{n} \pi^{m n}}{\prod_{k=1}^{n}(n-k) !(m-k) !}
$$

The proof is given in Appendix A.

Remark: Since the probability of two singular values being equal is zero, without loss of generality, in the theorem we consider strict inequalities in the ordering of the singular values. For a similar reason, we exclude the case $\left[V_{1, i}\right]=0$, and impose the normalization condition $\left[V_{1, i}\right]>0$ on every first element of the columns of $V$. This is in agreement with classical literature on this topic; see, e.g., [1] where strict inequalities in the ordering of the eigenvalues of symmetric matrices and a normalization condition on the eigenvectors matrix are considered. Notice that the strict ordering of the singular values, together with the normalization condition, make the mapping between $\Delta$ and $U, \Sigma, V$ one-to-one. This property is used in the proof of the theorem. In addition, we remark that in the literature the uniform density over the unitary group is known as the Haar invariant distribution [1]. The Haar invariant distribution is the only distribution with the property that if $U$ is distributed according to Haar, then $Q U \sim U$ for any unitary $Q$. The relation (12) therefore states that $U \in \mathbb{C}^{n, n}$ is distributed according to the Haar invariant distribution for the unitary group. Similarly, the uniform density over the normalized unitary matrices is known as the conditional Haar distribution.

The following theorem studies the case when $\Delta$ is a real matrix.

Theorem 2: Let $\Delta \in \mathbb{R}^{n, m}, m \geq n \geq 2$, be factored as in (4), with $\sigma_{1}>\sigma_{2}>\cdots>\sigma_{n}>0$, and $[V]_{1, i}>0$, for $i=1, \ldots, n$. The following statements are equivalent.

1) The p.d.f. $f_{\Delta}(\Delta)$ is unitarily invariant, i.e., $f_{\Delta} \in \mathcal{F}_{\mathcal{I}}$.

2) The joint p.d.f. of $U, \Sigma$, and $V$ is

$$
f_{U, \Sigma, V}(U, \Sigma, V)=f_{U}(U) f_{\Sigma}(\Sigma) f_{V}(V)
$$

where

$$
\begin{aligned}
& f_{U}(U)=\mathcal{U}\left[\left\{U: U^{T} U=I\right\}\right] \\
& f_{\Sigma}(\Sigma)=\Upsilon_{\mathbb{R}} f_{\Delta}(\Sigma) \prod_{i=1}^{n} \sigma_{i}^{m-n} \prod_{1 \leq i<k \leq n}\left(\sigma_{i}^{2}-\sigma_{k}^{2}\right) \\
& f_{V}(V)=\mathcal{U}\left[\left\{V: V^{T} V=I,[V]_{1, i}>0, i=1, \ldots, n\right\}\right]
\end{aligned}
$$


and $\Upsilon_{\mathbb{R}}$ is a normalization constant given by

$\Upsilon_{\mathbb{R}}=\frac{(8 \pi)^{n(m-1) / 2}}{2^{n(m+1) / 2}} \prod_{k=1}^{n} \frac{\Gamma\left(\frac{k-1}{2}\right)}{\Gamma(k-1)} \prod_{k=m-n+1}^{m} \frac{\Gamma\left(\frac{k-1}{2}\right)}{\Gamma(k-1)}$

The proof is given in Appendix B.

Remark: Similar comments to those of the complex case can be made regarding the distribution of the matrices $U$ and $V$. We remark that Theorem 2 is a generalization of the results reported in [1] and [27], which deal with the special case of real and symmetric matrices. Related results can also be found in [20] and [23].

In the next sections, we provide algorithms, based on Theorems 1 and 2, for the generation of uniform samples of $\Delta \epsilon$ $\mathbb{F}^{n, m}$ in the spectral norm ball of unit radius $\mathcal{B}_{\sigma}(1)$. We notice that uniform samples in $\mathcal{B}_{\sigma}(\rho)$ are simply obtained multiplying by $\rho$ the samples generated uniformly in $\mathcal{B}_{\sigma}(1)$. As discussed in [9] and [10], any radially symmetric density in the spectral norm may be generated starting from the uniform density on $\mathcal{B}_{\sigma}(1)$. The relation between the uniform density and a generic radially symmetric density on the support $\mathcal{B}_{\sigma}(\rho)$ is given in the following lemma.

Lemma 1: Let $\Delta \in \mathbb{F}^{n, m}$ be a random matrix with uniform distribution over $\mathcal{B}_{\sigma}(1)$, and let $z \in \mathbb{R}$ be an independent random variable with p.d.f. $f_{z}(z)$ over the support $[0, \rho]$. Define the random matrix

$$
\tilde{\Delta} \doteq \frac{z}{\bar{\sigma}(\Delta)} \Delta
$$

Then, the p.d.f. $f_{\tilde{\Delta}}(\tilde{\Delta})$ is radially symmetric in $\mathcal{B}_{\sigma}(\rho)$, i.e., $f_{\tilde{\Delta}}(\tilde{\Delta})=f_{\tilde{\Delta}}(r)$, where $r \doteq \bar{\sigma}(\tilde{\Delta}), r \in[0, \rho]$, and in particular

$$
f_{\tilde{\Delta}}(\tilde{\Delta})=\frac{f_{z}(r)}{\operatorname{vol}\left(\mathcal{B}_{\sigma}(\rho)\right) r^{d-1} d}
$$

where $d$ is the dimension of $\mathbb{F}^{n, m}$.

A proof of this lemma may be found in [9] and [10].

\section{UNIFORM SAMPLE GENERATION: COMPLEX CASE}

In this section, we show how to generate the samples of complex $\Delta$, by first generating the samples of the SVD factors $U, \Sigma$, $V$ according to their respective densities, and then constructing $\Delta=U \Sigma V^{*}$. We first concentrate on the generation of the singular values of full block matrices $\Delta \in \mathbb{C}^{n, m}$.

\section{A. Generation of the Singular Values}

Assume that the matrix $\Delta \in \mathbb{C}^{n, m}$ is uniformly distributed over the set $\mathcal{B}_{\sigma}(1)$, see (3). Then from Theorem 1 the p.d.f. of $\sum$ is

$$
\begin{gathered}
f_{\Sigma}(\Sigma)=K_{\mathbb{C}} \prod_{i=1}^{n} \sigma_{i}^{2(m-n)+1} \prod_{\substack{1 \leq i<k \leq n \\
1 \geq \sigma_{1}>\sigma_{2}>\cdots>\sigma_{n}>0}}\left(\sigma_{i}^{2}-\sigma_{k}^{2}\right)^{2}, \\
1 \geq
\end{gathered}
$$

with

$$
K_{\mathbb{C}}=\frac{\Upsilon_{\mathbb{C}}}{\operatorname{vol}\left(\mathcal{B}_{\sigma}(1)\right)}
$$

The value of the normalization constant $K_{\mathbb{C}}$ is related to the solution of a multiple integral known as the Selberg integral (see [27] and [35] for details on the derivation), and is given by

$$
K_{\mathbb{C}}=2^{n} \prod_{i=0}^{n-1} \frac{\Gamma(m+i+1)}{\Gamma^{2}(i+1) \Gamma(i+m-n+1)} .
$$

We remark that from (22), using (15) and (23), we can compute in closed form the volume $\operatorname{vol}\left(\mathcal{B}_{\sigma}(1)\right)$ of the complex spectral ball of unit radius.

For our subsequent developments, it is convenient to remove the ordering condition on the singular values. The so-obtained unordered p.d.f. is given by

$$
f_{\Sigma}^{(d)}(\Sigma)=\frac{K_{\mathbb{C}}}{n !} \prod_{i=1}^{n} \sigma_{i}^{2(m-n)+1} \prod_{1 \leq i<k \leq n}\left(\sigma_{i}^{2}-\sigma_{k}^{2}\right)^{2}
$$

where $0<\sigma_{i} \leq 1, i=1, \ldots, n$. The factorial term in the above equation is simply obtained observing that the ordered case is one of the $n$ ! possible permutations of the $n$ unordered singular values. We now introduce the change of variables

$$
x_{i}=\sigma_{i}^{2}, \quad i=1,2, \ldots, n .
$$

Applying the rule of change of variables for probability densities (68) in Appendix F, the p.d.f. $f_{x}$ of the random vector $x=$ $\left[x_{1}, x_{2}, \ldots, x_{n}\right]^{T}$ is given by

$$
f_{x}(x)=K_{x} \prod_{i=1}^{n} x_{i}^{m-n} \prod_{1 \leq i<k \leq n}\left(x_{i}-x_{k}\right)^{2}
$$

where

$$
K_{x} \doteq \frac{K_{\mathbb{C}}}{n ! 2^{n}}
$$

We remark that $f_{x}(x)$ can be written in terms of the determinant of a Vandermonde matrix. Given a vector $x=\left[x_{1}, x_{2}, \ldots, x_{n}\right]^{T}$, we let

$$
\mathcal{X}_{i}=\mathcal{X}_{i}\left(x_{i}\right) \doteq\left[\begin{array}{c}
1 \\
x_{i} \\
x_{i}^{2} \\
\vdots \\
x_{i}^{n-1}
\end{array}\right], \quad i=1, \ldots, n
$$

and, for $i=1, \ldots, n$, we define the truncated Vandermonde matrix $\mathcal{V}_{i}$ as

$$
\mathcal{V}_{i}=\mathcal{V}_{i}\left(x_{1}, x_{2}, \ldots, x_{i}\right) \doteq\left[\mathcal{X}_{1}, \mathcal{X}_{2}, \ldots, \mathcal{X}_{i}\right]
$$

Clearly, $\mathcal{V}_{i}$ depends only on the variables $x_{1}, x_{2}, \ldots, x_{i}$, while $\mathcal{V}_{n}$ is the standard Vandermonde matrix associated with the vector $x$. Then, by well-known properties of Vandermonde determinants, we write $f_{x}(x)$ as

$$
f_{x}(x)=K_{x}\left|\mathcal{V}_{n}\right|^{2} \prod_{i=1}^{n} x_{i}^{m-n}
$$


We now concentrate on the generation of random samples distributed according to (27).

A standard method for random generation with multivariate distributions is the so-called conditional method [17], [34]. The basic idea of this method is to generate the first random variable, then generate the next one conditional on the first one, and so forth. In other words, the conditional method reduces an n-dimensional generation problem to $n$ one-dimensional problems. However, it requires the computation of the marginal densities, which is often a very difficult task [18]. For the sake of completeness, the conditional method is recalled below.

The Conditional Method: A joint p.d.f. $f_{x}(x)$ can be written as

$$
f_{x}\left(x_{1}, \ldots, x_{n}\right)=f_{1}\left(x_{1}\right) f_{2}\left(x_{2} \mid x_{1}\right) \ldots f_{n}\left(x_{n} \mid x_{1} \ldots x_{n-1}\right)
$$

where $f_{i}\left(x_{i} \mid x_{1}, \ldots, x_{i-1}\right)$ are the conditional densities, defined as the ratio of marginal densities

$$
f_{i}\left(x_{i} \mid x_{1}, \ldots, x_{i-1}\right)=\frac{f_{i}\left(x_{1}, \ldots, x_{i}\right)}{f_{i-1}\left(x_{1}, \ldots, x_{i-1}\right)} .
$$

The marginal densities $f_{i}\left(x_{1}, \ldots, x_{i}\right)$ are defined as

$$
f_{i}\left(x_{1}, \ldots, x_{i}\right) \doteq \int \ldots \int f_{x}\left(x_{1}, \ldots, x_{n}\right) d x_{i+1} \ldots d x_{n}
$$

A vector $x$ with density $f_{x}(x)$ can therefore be obtained generating sequentially the $x_{i}$ 's, $i=1, \ldots, n$, where $x_{i}$ is distributed according to the univariate density $f_{i}\left(x_{i} \mid x_{1}, \ldots, x_{i-1}\right)$.

The following theorem and its corollary provide a closed form expression for the marginal densities of the multivariate density function $f_{x}(x)$ defined in (27). These results provide a direct computation of the conditional densities that are needed for the application of the conditional method.

Theorem 3: Let $f_{x}(x)$ be given by (27), then the marginal density

$$
f_{i}\left(x_{1}, \ldots, x_{i}\right)=\int_{0}^{1} \cdots \int_{0}^{1} f_{x}\left(x_{1}, \ldots, x_{n}\right) d x_{i+1} \ldots d x_{n}
$$

is equal to

$$
f_{i}\left(x_{1}, \ldots, x_{i}\right)=K_{x} \frac{(n-i) !}{|M|}\left|\mathcal{V}_{i}^{T} M \mathcal{V}_{i}\right| \prod_{k=1}^{i} x_{k}^{m-n}
$$

where $\mathcal{V}_{i}$ is defined in (26), and $M \doteq R^{-1}$, being $R$ a constant symmetric matrix defined as

$$
[R]_{r, \ell} \doteq \frac{1}{r+\ell+m-n-1}, \quad r, \ell=1, \ldots, n
$$

The proof of this theorem is reported in Appendix C.

Therefore, to compute the marginal density (31), one first needs to compute the determinant $\left|\mathcal{V}_{i}^{T} M \mathcal{V}_{i}\right|$. To this end, we now express this determinant separating the variables $x_{1}, \ldots, x_{i-1}$ from the variable $x_{i}$, as detailed in the following corollary.
Corollary 1: Let $x_{1}, x_{2}, \ldots, x_{i-1}$ be fixed, then the marginal density (31), evaluated in $x_{1}, x_{2}, \ldots, x_{i-1}$, is given by the polynomial in $x_{i}$

$$
p_{i}\left(x_{i}\right)=C_{i-1} x_{i}^{m-n} \sum_{k=0}^{2(n-1)} b_{k} x_{i}^{k} .
$$

The coefficients $b_{k}$ are given by

$$
\begin{aligned}
b_{k} & =b_{k}\left(x_{1}, \ldots, x_{i-1}\right) \\
& \doteq \sum_{\{r+\ell=k+2\}}\left[W_{i-1}\right]_{r, \ell}, \quad k=0, \ldots, 2(n-1)
\end{aligned}
$$

being

$$
C_{0}=\frac{K_{x}(n-1) !}{|M|}, \quad W_{0}=M
$$

and, for $i=2, \ldots, n$

$$
\begin{aligned}
C_{i-1} & =C_{i-1}\left(x_{1}, \ldots, x_{i-1}\right) \\
& \doteq \frac{K_{x}(n-i) !}{|M|}\left|Z_{i-1}\right| \prod_{k=1}^{i-1} x_{k}^{m-n} \\
W_{i-1} & =W_{i-1}\left(x_{1}, \ldots, x_{i-1}\right) \\
& \doteq M-M \mathcal{V}_{i-1} Z_{i-1}^{-1} \mathcal{V}_{i-1}^{T} M \\
Z_{i-1} & =Z_{i-1}\left(x_{1}, \ldots, x_{i-1}\right) \\
& \doteq \mathcal{V}_{i-1}^{T} M \mathcal{V}_{i-1} .
\end{aligned}
$$

Moreover, the following recursion holds:

$$
p_{i}\left(x_{i}\right)=p_{i-1}\left(x_{i-1}\right) \frac{1}{n-i+1} x_{i}^{m-n} \sum_{k=0}^{2(n-1)} b_{k} x_{i}^{k} .
$$

The proof of this result is reported in Appendix D. We now make a few remarks regarding Theorem 3 and Corollary 1 .

\section{Remarks:}

1) The contribution of Theorem 3 is to give a closed form solution for the multiple integral (30). In particular, in order to apply the conditional method, it is useful to express $f_{i}\left(x_{1}, \ldots, x_{i}\right)$ in the form of (32), where we separate the variables $x_{1}, x_{2}, \ldots, x_{i-1}$ from the variable $x_{i}$. In fact, at the $i$ th step of the conditional method, the variables up to $i-1$ are given, and only the dependence on $x_{i}$ is required. In this case, (32) represents a polynomial in the $x_{i}$ variable, whose coefficients $b_{k}$ and the multiplicative constant $C_{i-1}$ can be easily computed according to (33) and (34), once the values of $x_{1}, x_{2}, \ldots, x_{i-1}$ are known.

2) We observe that for $k \geq 2$ the quantities $\left|Z_{k}\right|$ and $Z_{k}^{-1}$ appearing in (34) and (35) can be computed recursively, so that no matrix inversion or determinant computation is required. In particular, we have

$$
Z_{k}=\mathcal{V}_{k}^{T} M \mathcal{V}_{k}=\left[\begin{array}{cc}
Z_{k-1} & \mathcal{V}_{k-1}^{T} M \mathcal{X}_{k} \\
\mathcal{X}_{k}^{T} M \mathcal{V}_{k-1} & \mathcal{X}_{k}^{T} M \mathcal{X}_{k}
\end{array}\right]
$$

Using the block matrix inversion formula, we obtain $\mathcal{Z}_{k}^{-1}$ as shown in the equation at the bottom of the next page, and

$$
\left|Z_{k}\right|=\delta_{k}\left|Z_{k-1}\right|
$$

where $\delta_{k}=\mathcal{X}_{k}^{T} W_{k-1} \mathcal{X}_{k}$ is a positive number. 
3) We notice that combining (28) and (37), a closed form expression for the conditional density can also be obtained

$$
f_{i}\left(x_{i} \mid x_{1}, \ldots, x_{i-1}\right)=K_{i} x_{i}^{m-n} \sum_{k=0}^{2(n-1)} b_{k}\left(x_{1}, \ldots, x_{i}\right) x_{i}^{k}
$$

where

$$
K_{i} \doteq \begin{cases}K_{x} \frac{(n-1) !}{|M|}, & \text { for } i=1 \\ (n-i+1)^{-1}, & \text { for } i=2, \ldots, n .\end{cases}
$$

An explicit algorithm for the generation of samples of the singular values distributed according to the p.d.f. (24) is now given.

Algorithm 1 (Singular Values Generation): A random vector $\sigma=\left[\sigma_{1}, \sigma_{2}, \ldots, \sigma_{n}\right]^{T}$ distributed according to (24) can be generated via the following algorithm.

\section{$\diamond$ Initialization.}

$\triangleright i \leftarrow 1 ; W_{0} \leftarrow M ;$ where $M$ is defined in Theorem 3 .

$\diamond$ Generation.

$\triangleright$ Let $b_{k} \leftarrow \sum_{\{r+\ell=k+2\}}\left[W_{i-1}\right]_{r, \ell} ; k=1, \ldots, 2(n-1)$.

$\triangleright$ Generate $x_{i}$ according to the

polynomial density

$$
f_{i}\left(x_{i} \mid x_{1}, \ldots, x_{i-1}\right)=K_{i} x_{i}^{m-n} \sum_{k=0}^{2(n-1)} b_{k} x_{i}^{k}
$$

$\diamond$ Check.

where $K_{i}$ is given by (38).

$$
\triangle \text { if } i=n \text { then goto [End]. }
$$

$\diamond$ Update.

$\triangleright$ Construct $\mathcal{X}_{i}=\left[1, x_{i}, \ldots, x_{i}^{n-1}\right]^{T}$ and

$\mathcal{V}_{i}=\left[\mathcal{X}_{1} \cdots \mathcal{X}_{i}\right]$

$\triangleright$ Let $\delta_{i} \leftarrow \mathcal{X}_{i}^{T} W_{i-1} \mathcal{X}_{i}$

$\triangleright$ If $i=1$ then $Z_{i} \leftarrow \delta_{i}$.

$\triangleright$ If $i>1$ then

$\triangleright Z_{i}^{-1} \leftarrow$

$$
\left[\begin{array}{c}
Z_{i-1}^{-1}+Z_{i-1}^{-1} \mathcal{V}_{i-1}^{T} M \mathcal{X}_{i} \mathcal{X}_{i}^{T} M \mathcal{V}_{i-1} Z_{i-1}^{-1} / \delta_{i} \\
-\left(Z_{i-1}^{-1} \mathcal{V}_{i-1}^{T} M \mathcal{X}_{i}\right)^{T} / \delta_{i} \\
-Z_{i-1}^{-1} \mathcal{V}_{i-1}^{T} M \mathcal{X}_{i} / \delta_{i} \\
1 / \delta_{i}
\end{array}\right] ;
$$

$\triangleright\left|Z_{i}\right| \leftarrow \delta_{i}\left|Z_{i-1}\right|$.

$\checkmark$ Loop.

$$
\triangleright W_{i} \leftarrow M-M \mathcal{V}_{i} Z_{i}^{-1} V_{i}^{T} M .
$$

$\diamond$ End.

$$
\triangleright i \leftarrow i+1 \text {; goto [Generation]. }
$$

$\triangleright$ Return $\sigma=\left[\sqrt{x_{1}}, \ldots, \sqrt{x_{n}}\right]^{T}$.
In this algorithm, each $\sigma_{i}$ is generated according to a univariate polynomial density. Standard and efficient algorithms for the generation of samples distributed according to a given polynomial density are available in the literature. Among these techniques we recall the classical inversion method [17], [34].

\section{B. Generation of Haar Samples}

In this subsection, we concentrate on the generation of the samples of $U$ and $V$ according to the distributions (12) and (14).

We first consider the problem of generating uniform Haar samples of unitary $n \times n$ matrices. From the discussion following Theorem 1, we have that the distribution of $U$ must be the same of the distribution of $W U$, for any given unitary matrix $W$. Consider a random matrix $X \in \mathbb{C}^{n, n}=\operatorname{Re}(X)+j \operatorname{Im}(X)$, such that the elements of $\operatorname{Re}(X)$ and $\operatorname{Im}(X)$ are independent and normally distributed with zero mean and variance equal to one. The invariance property of the normal distribution under unitary transformations implies that, for any unitary matrix $W$, the distribution of $W X$ is the same as the distribution of $X$. Now, we let $X=Q R$ be the $Q R$ factorization of $X$, where the diagonal entries of $R$ are set to be real and positive in order to make the representation unique. Then, as $W X \sim X$, it follows that $W Q \sim Q$; that is, $Q$ is distributed according to the Haar invariant distribution.

This discussion suggests the following simple algorithm for the generation of samples from the Haar invariant distribution.

Algorithm 2 (Generation of Haar Samples): A random unitary matrix $H \in \mathbb{C}^{q, q}$ distributed according to (12) can be generated via the following algorithm.

$\diamond$ Build $X$.

$\triangleright$ Construct $X^{\Re}, X^{\Im} \in \mathbb{R}^{q, q}$, where each entry of $X^{\Re}$ and $X^{\Im}$ is an

independent gaussian variable with zero mean and variance equal to one.

$\diamond \mathbf{Q R}$. $\triangleright X \leftarrow X^{\Re}+j X^{\Im}$.

$\triangleright[Q, R] \leftarrow Q R(X)$. $\triangleright Q \leftarrow Q \operatorname{diag}\left(e^{-j \phi_{1}}, e^{-j \phi_{2}}, \ldots, e^{-j \phi_{q}}\right)$, where $\phi_{i}$ is the phase of the $(i, i)$ element of $R$.

$\diamond$ End. Return $H \leftarrow Q$.

This algorithm is one of the simplest methods for the generation of uniform unitary matrices. Other known methods are based, for example, on products of elementary Euler transformations; see, e.g., [44].

We remark that the Haar distribution may be introduced also for rectangular matrices $X \in \mathbb{C}^{m, n}$ having orthogonal columns. In this case, it can be observed that uniform samples of $X$ can

$$
Z_{k}^{-1}=\left[\begin{array}{cc}
Z_{k-1}^{-1}+Z_{k-1}^{-1} \mathcal{V}_{k-1}^{T} M \mathcal{X}_{k} \mathcal{X}_{k}^{T} M \mathcal{V}_{k-1} Z_{k-1}^{-1} / \delta_{k} & -Z_{k-1}^{-1} \mathcal{V}_{k-1}^{T} M \mathcal{X}_{k} / \delta_{k} \\
-\left(Z_{k-1}^{-1} \mathcal{V}_{k-1}^{T} M \mathcal{X}_{k}\right)^{T} / \delta_{k} & 1 / \delta_{k}
\end{array}\right]
$$


be obtained from uniform samples of a square $m \times m$ unitary matrix, neglecting the last $m-n$ columns. This fact follows from properties of the unitary group; see for instance [23, Ch. 5].

We next consider the generation of conditional Haar samples of $m \times n$ matrices $V$. Notice first that the normalization condition $[V]_{1, i}>0$ may be written as $V=\tilde{V} D$, where $\tilde{V} \in \mathbb{C}^{m, n}$ is a (nonnormalized) unitary matrix, and $D$ is a diagonal unitary matrix $D=\operatorname{diag}\left(e^{-j \theta_{1}}, \ldots, e^{-j \theta_{n}}\right)$, where $\theta_{i}$ is the phase of $[\tilde{V}]_{1, i}$. Using a technique similar to the one in the proof of Theorem 1, it can be shown that if $\tilde{V}$ is distributed according to the Haar distribution, then $V$ is distributed according to the conditional Haar distribution. Samples of matrices drawn from the latter distribution may therefore be obtained normalizing the samples drawn from the Haar distribution.

\section{UNIFORM SAMPLE GENERATION: REAL CASE}

We consider first the generation of the singular values of full block matrices $\Delta \in \mathbb{R}^{n, m}$ and then the generation of $U$ and $V$.

\section{A. Generation of the Singular Values}

Assume that the matrix $\Delta \in \mathbb{R}^{n, m}$ is uniformly distributed over the set $\mathcal{B}_{\sigma}(1)$; see (3). Then, from Theorem 2 the p.d.f. of $\Sigma$ is

$$
\begin{array}{r}
f_{\Sigma}(\Sigma)=K_{\mathbb{R}} \prod_{i=1}^{n} \sigma_{i}^{m-n} \prod_{\substack{1 \leq i<k \leq n \\
1 \geq \sigma_{1}>\sigma_{2}>\cdots>\sigma_{n}>0}}\left(\sigma_{i}^{2}-\sigma_{k}^{2}\right) \\
1
\end{array}
$$

with

$$
K_{\mathbb{R}}=\frac{\Upsilon_{\mathbb{R}}}{\operatorname{vol}\left(\mathcal{B}_{\sigma}(1)\right)}
$$

The normalization constant $K_{\mathbb{R}}$ is related to the solution of the Selberg integral, and is given by

$$
\begin{aligned}
& K_{\mathbb{R}}=n ! \pi^{n / 2} \\
& \quad \prod_{i=0}^{n-1} \frac{\Gamma\left(1+\frac{m+i}{2}\right)}{\Gamma\left(\frac{3}{2}+\frac{i}{2}\right) \Gamma\left(\frac{m-n+i+1}{2}\right) \Gamma\left(1+\frac{i}{2}\right)}
\end{aligned}
$$

We remark that from (40), using (20) and (41), we compute in closed form the volume of the real spectral ball of unit radius.

In this case, the closed form computation of the multiple integrals needed for the application of the conditional method cannot be performed with the techniques developed for the complex case. In this section, we develop an algorithm based on the rejection method from a dominating density; see [17]. First, we find an appropriate bounding function for the probability density of the singular values. It can be shown that (39) is bounded from above as follows

$$
f_{\Sigma}(\Sigma) \leq K_{\mathbb{R}} \prod_{i=1}^{n} \sigma_{i}^{2(n-i)+m-n}
$$

In order to decouple the domain of the variables of the bounding density, we introduce the change of variable

$$
x_{i}=\frac{\sigma_{i}}{\sigma_{i-1}}
$$

for $i=1, \ldots, n$ and $\sigma_{0} \doteq 1$. Notice that the domain of the new variables $x_{i}$ is the interval $(0,1]$ for $i=1, \ldots, n$. The inverse transformation is given by

$$
\sigma_{i}(x)=\prod_{k=1}^{i} x_{k} .
$$

Applying the rule of change of variables for probability densities (68) in Appendix F, the p.d.f. $f_{x}$ of the vector $x=\left[x_{1}, x_{2}, \ldots, x_{n}\right]^{T}$ is

$$
f_{x}(x)=f_{\Sigma}\left(\sigma_{1}(x), \ldots, \sigma_{n}(x)\right) \prod_{i=1}^{n} x_{i}^{n-i} .
$$

Using (42), we obtain a bounding function for $f_{x}(x)$

$$
f_{x}(x) \leq \gamma \bar{f}_{x}(x)
$$

where $\bar{f}_{x}(x)$ is the dominating probability density, given by

$$
\bar{f}_{x}(x)=\prod_{i=1}^{n}\left(1+\beta_{i}\right) x_{i}^{\beta_{i}}
$$

and $\beta_{i}=(m+n)(n-i+1)-n(n+1)+i(i-1)+n-i ; i=$ $1, \ldots, n$. The constant $\gamma$ is given by

$$
\gamma=\frac{K_{\mathbb{R}}}{\prod_{i=1}^{n}\left(1+\beta_{i}\right)}
$$

We remark that the density function $\bar{f}_{x}(x)$ is such that the $x_{i}$ 's are independent. Therefore, the generation of a random vector $x$ distributed according to $\bar{f}_{x}(x)$ may be efficiently performed according to the techniques described in [17]. We report below an algorithm for the generation of random samples of the vector $\sigma$, which is based on $n$-dimensional rejection from the dominating density $\bar{f}_{x}(x)$.

Algorithm 3 (Singular Values Generation): A random vector $\sigma=\left[\sigma_{1}, \sigma_{2}, \ldots, \sigma_{n}\right]^{T}$ distributed according to (39) can be generated via the following algorithm.

$\diamond$ Generation.

$\triangleright$ For $i=1, \ldots, n:$ Generate $w_{i} \sim \mathcal{U}[[0,1]]$, and compute $x_{i}=w^{(1 / 1+\beta)}$.

$\checkmark$ Computation of $\boldsymbol{t}$. $\triangleright t \leftarrow \gamma\left(\bar{f}_{x}(x) / f_{x}(x)\right)$ with $\bar{f}_{x}(x)$ given by (46) and $f_{x}(x)$ given by (44).

\section{$\diamond$ Rejection.} $\triangleright$ Generate $u \sim \mathcal{U}[[0,1]]$.

$\triangleright$ If $u t \leq 1$, compute $\sigma(x)$ according to (43) and return $\sigma$. $\triangle$ Else goto [Generation].

It can be shown [17] that the expected value of the rejection rate, i.e., the expected number of iterations needed to produce a valid vector, is given by $\gamma$. This fact can be used to compare the 
TABLE II

COMPARISON OF REJECTION RATES FOR SQUARE REAL MATRICES

\begin{tabular}{c|ccccccc}
\hline$n$ & 2 & 3 & 4 & 5 & 6 & 7 & 8 \\
\hline$\gamma$ & 1.5 & 5 & 43.75 & 1102 & 84893 & $2 \mathrm{e} 7$ & $1 \mathrm{e} 10$ \\
\hline$\gamma_{F}$ & 3 & 26.7 & 640 & $4 \mathrm{e} 4$ & $6 \mathrm{e} 6$ & $2 \mathrm{e} 9$ & $2 \mathrm{e} 12$ \\
\hline
\end{tabular}

rejection rate $\gamma$ of Algorithm 3 with the one obtained by simply overbounding the set $\mathcal{B}_{\sigma}(1)$ with the Frobenius ball $\mathcal{B}_{F}(\sqrt{n})$. The two rejection rates $\gamma$ and $\gamma_{F}$ are compared in Table II, for square real matrices of order $n$. From Table II, we see that the proposed algorithm improves upon the standard rejection from the Frobenius norm ball. We notice, however, that the growth is still nonpolynomial and this is in contrast with the algorithm derived for the complex case, which is polynomial-time. Further research is therefore needed to derive a result similar to Theorem 3 for the computation of the marginal densities in closed form; see the new framework proposed in [12] for the solution of the real case.

Next, we present an algorithm similar to Algorithm 2, for the generation of orthogonal matrices $H$ distributed according to the Haar density. This algorithm is based on the QR decomposition and is reported also in [36].

\section{B. Generation of Haar Samples}

Algorithm 4 (Generation of Haar Samples): A random orthogonal matrix $H \in \mathbb{R}^{q, q}$ distributed according to (12) can be generated via the following algorithm.

$\diamond$ Generation of $\boldsymbol{X}$.

$\triangleright$ Construct $X \in \mathbb{R}^{q, q}$, where each entry of $X$ is an independent gaussian variable with zero mean and variance equal to one.

$\triangleright[Q, R] \leftarrow Q R(X)$.

$\triangleright Q \leftarrow Q \operatorname{diag}\left(s_{1}, s_{2}, \ldots, s_{q}\right)$, where $s_{i}$ is the sign of the $(i, i)$ element of $R$.

$\diamond$ End. Return $H \leftarrow Q$.

\section{NUMERICAL EXAMPLE}

We considered an example concerning a five masses springdamper model with parametric uncertainty on the stiffness and damping parameters, and complex uncertainty due to unmodeled dynamics [8]. This flexible structure may be modeled as an $M-\Delta$ configuration, with $M(s)=C(s I-A)^{-1} B+D$, where the matrices $A, B$, and $C$ are given in Appendix $\mathrm{H}$, and $D=0$. The matrix $\Delta$ consists of two repeated real parameters $q_{1}, q_{2}$ and one full complex block $\Delta_{1} \in \mathbb{C}^{4,4}$

$$
\Delta=\left[\begin{array}{ccc}
q_{1} I_{5} & 0 & 0 \\
0 & q_{2} I_{5} & 0 \\
0 & 0 & \Delta_{1}
\end{array}\right]
$$

For this $M-\Delta$ system, lower and upper bounds $1 / \mu^{+}$and $1 / \mu^{-}$ of the robustness margin $1 / \mu$ have been computed with the Matlab $\mu$-Analysis and Synthesis Toolbox obtaining

$$
1 / \mu^{+} \approx 1 / \mu^{-} \approx 0.394
$$

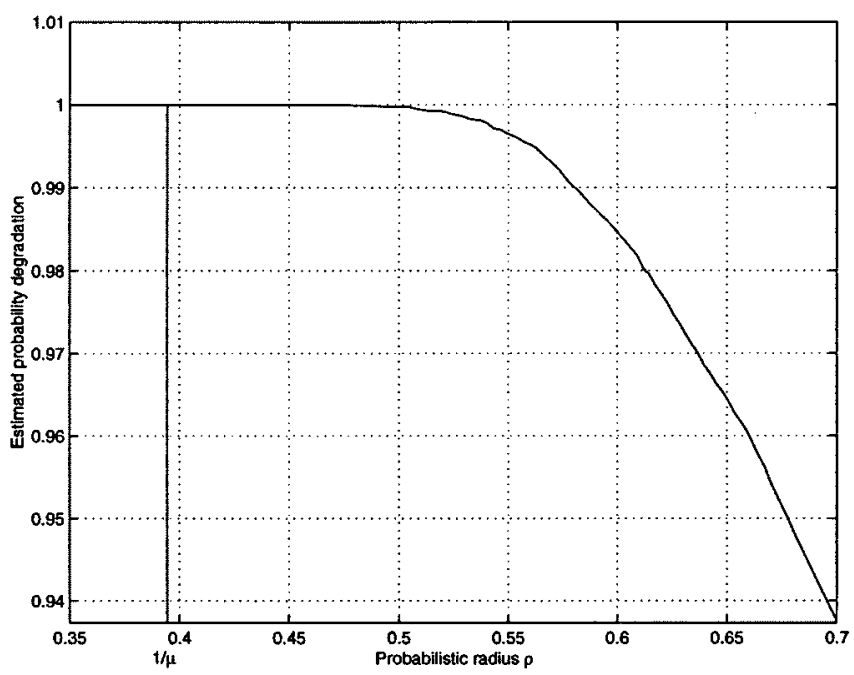

Fig. 1. Estimated probability degradation function $\hat{p}(\rho)$.

Taking $\epsilon=\delta=0.01$, by means of the Chernoff bound, we obtained $N \geq 26492$. Subsequently, for this sample size, using the algorithms given in Section IV, we estimated the probability degradation function $p(\rho)$ for 100 values of $\rho$ in the range $[0.35,0.70]$. This plot is shown in Fig. 1 together with the lower bound of the deterministic robustness margin. From this plot, we observe that if a $2 \%$ loss of probabilistic performance may be tolerated, then the stability margin may be increased of approximately $56 \%$ with respect to its deterministic counterpart. In fact, the risk adjusted stability margin for $p^{*}=0.98$ is $\rho(0.98) \approx 0.62$. In addition we notice that the estimated probability is equal to one up to $\rho \approx 0.47$. We conclude that in this example the upper and lower bounds of $\mu$ approximately coincide, so that $1 / \mu$ is a nonconservative deterministic measure of robustness but this measure turns out to be quite conservative in a probabilistic sense.

From the computational point of view, the proposed probabilistic algorithm is polynomial-time. The computational cost is proportional to the cost of generating one sample of uncertainty, and a crude Matlab implementation of the algorithms in Section IV required about $2 \times 10^{4}$ flops for generating one sample of $\Delta_{1} \in \mathbb{C}^{4,4}$.

\section{CONCLUSION}

In this paper, we studied randomized algorithms for robustness analysis in the presence of general uncertainty structures. This approach may be used in conjunction with standard worst-case techniques, in order to obtain additional information about the probabilistic degradation of system performance when the uncertainty level goes beyond the deterministic margin. In particular, polynomial-time algorithms for the generation of matrix samples have been presented for the complex case. For the real case, a rejection algorithm was proposed, while a different framework for a closed form solution has been introduced in [12].

Current research is directed toward the extension of the proposed approach to the synthesis of probabilistic robust 
controllers and to the development of probabilistic optimization algorithms.

\section{APPENDIX}

\section{A. Proof of Theorem 1}

Consider the transformation given by the SVD of $\Delta=U \Sigma V^{*}$, defined in (4). The strict inequalities in the ordering of the singular values, together with the imposed normalization conditions on the columns of $V$, make the mapping between $\Delta$ and $U, \Sigma, V$ one-to-one; see for instance [22, Section 7.3]. The joint p.d.f. in the new variables $U, \Sigma, V$ may be obtained applying the rule (68) in Appendix F,

$$
f_{U, \Sigma, V}(U, \Sigma, V)=f_{\Delta}(\Sigma) J(U, \Sigma, V ; \Delta)
$$

The differential of $\Delta$ is given by

$$
d \Delta=d U \Sigma V^{*}+U d \Sigma V^{*}+U \Sigma d V^{*} .
$$

If $m>n$, let $V_{1} \in \mathbb{C}^{m, m-n}$ be such that $\bar{V} \doteq\left[V \mid V_{1}\right]$ is unitary, otherwise if $m=n$ let $\bar{V} \equiv V$. Then, multiplying (49) by $U^{*}$ on the left and by $\bar{V}$ on the right, we obtain

$$
Z \doteq U^{*} d \Delta \bar{V}=\left[U^{*} d U \Sigma \mid 0\right]+[d \Sigma \mid 0]+\Sigma d V^{*} \bar{V}
$$

Now, using the rule G.2 in Appendix G, we have that $J(U, \Sigma, V ; \Delta)=J(d U, d \Sigma, d V ; d \Delta)$, and applying the chain rule for Jacobians G.1, we have further

$$
J(d U, d \Sigma, d V ; d \Delta)=J(d U, d \Sigma, d V ; Z) J(Z ; d \Delta)
$$

Since by G.5 $J(Z ; d \Delta)=1$, we have that the Jacobian we are interested in is equal to

$$
J(U, \Sigma, V ; \Delta)=J(d U, d \Sigma, d V ; Z)
$$

Next, we rewrite (50) in the form

$$
Z=\left[S_{u} \Sigma \mid 0\right]+[d \Sigma \mid 0]+\Sigma \bar{S}_{v}^{*}
$$

where

$$
S_{u} \doteq U^{*} d U \in \mathbb{C}^{n, n}, \quad \bar{S}_{v} \doteq \bar{V}^{*} d V \in \mathbb{C}^{m, n} .
$$

Since, by G.5, the Jacobian $J\left(d U, d \Sigma, d V ; S_{u}, d \Sigma, \bar{S}_{v}\right)$ is equal to one, applying again the chain rule, we have

$$
\begin{aligned}
& J(d U, d \Sigma, d V ; Z) \\
& \quad=J\left(d U, d \Sigma, d V ; S_{u}, d \Sigma, \bar{S}_{v}\right) J\left(S_{u}, d \Sigma, \bar{S}_{v} ; Z\right) \\
& \quad=J\left(S_{u}, d \Sigma, \bar{S}_{v} ; Z\right) .
\end{aligned}
$$

We now concentrate on the evaluation of $J\left(S_{u}, d \Sigma, \bar{S}_{v} ; Z\right)$. First, notice that $S_{u}$ is skew-Hermitian. This is easily seen by differentiating the identity $U^{*} U=I$, obtaining $d U^{*} U+U^{*} d U=0$. Similarly, if $m>n, \bar{S}_{v}$ can be partitioned as

$$
\bar{S}_{v}=\left[\begin{array}{l}
S_{v} \\
Q^{*}
\end{array}\right]
$$

where $S_{v} \doteq V^{*} d V \in \mathbb{C}^{n, n}$ is skew-Hermitian, and $Q \doteq$ $d V^{*} V_{1} \in \mathbb{C}^{n, m-n}$. The matrix $Z$ is finally rewritten in the form

$$
Z=\left[S_{u} \Sigma-\Sigma S_{v}+d \Sigma \mid \Sigma Q\right]
$$

Clearly, if $m=n$, then $\bar{S}_{v} \equiv S_{v}$ and $Z=\left[S_{u} \Sigma-\Sigma S_{v}+d \Sigma\right]$.

Let now examine the number of free variables that describe the quantities of interest. The matrix $\Delta$ is described by means of $2 n m$ real variables. The unitary matrix $U$ is described by means of $n_{u} \doteq n^{2}$ real variables, $\Sigma$ is described by means of its $n$ diagonal entries, therefore $V$ is described by the remaining $n_{v} \doteq$ $2 n m-n^{2}-n$ real variables. Since an $m \times n$ complex matrix with orthogonal columns is described by $2 n m-n^{2}$ variables [23], we notice that the normalization imposed in (4) on the columns of $V$ fixes $n$ of the free variables. The differentials $d U, d V, d \Sigma$ are described by the same number of free variables as $U, V$, and $\Sigma$. Therefore, $S_{u}$ and $\bar{S}_{v}^{*}=\left[S_{v}^{*} \mid Q\right]$ are described by $n_{u}$ and $n_{v}$ variables, respectively. Since $d \Sigma$ is real diagonal, we choose its $n$ free variables as the diagonal entries $\eta_{i}=d \sigma_{i}, 1 \leq i \leq n$. Since $S_{u}$ is skew-Hermitian, we choose the $n_{u}$ free variables $\mu_{i k}^{\Re}, \mu_{i k}^{\Im}$ as the coefficients of the standard orthonormal basis of the space of $n \times n$ skew-Hermitian matrices. In particular

$$
S_{u}=\sum_{1 \leq i<k \leq n}\left(\mu_{i k}^{\Re} B_{i k}^{\Re}+\mu_{i k}^{\Im} B_{i k}^{\Im}\right)+\sum_{i=1}^{n} \mu_{i i}^{\Im} D_{i}
$$

where $B_{i k}^{\Re}, B_{i k}^{\Im}$, and $D_{i}$ are the elements of the basis. Denoting by $E_{i k}$ an $n \times n$ matrix having one in position $(i, k)$ and zero otherwise, the elements of the basis are defined as

$$
\begin{array}{rlrl}
B_{i k}^{\Re} & \doteq \frac{1}{\sqrt{2}}\left(E_{i k}-E_{k i}\right), & 1 \leq i<k \leq n \\
B_{i k}^{\Im} & \doteq \frac{j}{\sqrt{2}}\left(E_{i k}+E_{k i}\right), & 1 \leq i<k \leq n \\
D_{i} \doteq j E_{i i}, \quad 1 \leq i \leq n . &
\end{array}
$$

Similarly, considering that the matrix $\bar{S}_{v}=\left[S_{v}^{*} \mid Q\right]^{*}$ is the first (block) column of the $(m \times m)$ skew-Hermitian matrix $\bar{V}^{*} d \bar{V}$, we choose $n^{2}-n$ free variables $\nu_{i k}^{\Re}, \nu_{i k}^{\Im}$ such that

$$
S_{v}=\sum_{1 \leq i<k \leq n}\left(\nu_{i k}^{\Re} B_{i k}^{\Re}+\nu_{i k}^{\Im} B_{i k}^{\Im}\right)+\sum_{i=1}^{n} h_{i}\left(\nu^{\Re}, \nu^{\Im}\right) D_{i}
$$

where $h_{i}\left(\nu^{\Re}, \nu^{\Im}\right)$ is some function of the variables $\nu^{\Re}, \nu^{\Im}$. The remaining $n_{v}-\left(n^{2}-n\right)=2 n(m-n)$ free variables, $q_{i k}^{\Re}, q_{i k}^{\Im}$ are needed to describe $Q$

$$
[Q]_{i k}=\frac{1}{\sqrt{2}}\left(q_{i k}^{\Re}+j q_{i k}^{\Im}\right), \quad 1 \leq i \leq n ; 1 \leq k \leq m-n
$$


The $(i, k)$ element of $Z$ may now be expressed as

$$
[Z]_{i k}=\left\{\begin{array}{c}
\left(\mu_{i k}^{\Re}+j \mu_{i k}^{\Im}\right) \sigma_{k} / \sqrt{2}-\left(\nu_{i k}^{\Re}+j \nu_{i k}^{\Im}\right) \sigma_{i} / \sqrt{2} \\
1 \leq i<k \leq n \\
\left(-\mu_{k i}^{\Re}+j \mu_{k i}^{\Im}\right) \sigma_{k} / \sqrt{2}+\left(\nu_{k i}^{\Re}-j \nu \Im\right) \sigma_{i}^{\Im} / \sqrt{2} \\
1 \leq k<i \leq n \\
\eta_{i}+j \mu_{i i}^{\Im} \sigma_{i}-j h_{i}\left(\nu^{\Re}, \nu^{\Im}\right) \sigma_{i} \\
i=k, 1 \leq i \leq n \\
\left(q_{i r}^{\Re}+j q_{i r}^{\Im}\right) \sigma_{i} / \sqrt{2} \\
r=k-n ; 1 \leq i \leq n ; n<k \leq m .
\end{array}\right.
$$

To compute the Jacobian $J\left(S_{u}, d \Sigma, \bar{S}_{v} ; Z\right)$, we construct the scheme of partial derivatives shown at the bottom of the page, where where

$$
\begin{gathered}
C=\frac{1}{\sqrt{2}} \operatorname{blockdiag}\left(\operatorname{diag}\left(\sigma_{2}, \ldots, \sigma_{n}\right), \operatorname{diag}\left(\sigma_{3}, \ldots, \sigma_{n}\right), \ldots,\right. \\
\left.\operatorname{diag}\left(\sigma_{n-1}, \sigma_{n}\right), \sigma_{n}\right) \\
D=\frac{1}{\sqrt{2}} \operatorname{blockdiag}\left(\sigma_{1} I_{n-1}, \sigma_{2} I_{n-2}, \ldots, \sigma_{n-2} I_{2}, \sigma_{n-1}\right) \\
F=\frac{1}{\sqrt{2}} \operatorname{blockdiag}\left(\sigma_{1} I_{m-n}, \sigma_{2} I_{m-n}, \ldots, \sigma_{n-1} I_{m-n},\right. \\
\left.\sigma_{n} I_{m-n}\right) .
\end{gathered}
$$

The matrix of partial derivatives is block triangular, therefore the matrices $H^{\Re}, H^{\Im}$ do not affect the value of its determinant, which is given by

$$
J\left(S_{u}, d \Sigma, \bar{S}_{v} ; Z\right)=|\Sigma||F|^{2}\left|\begin{array}{rr}
C & -D \\
-D & C
\end{array}\right|\left|\begin{array}{rr}
C & D \\
-D & -C
\end{array}\right| .
$$

Using Schur complement, we have that

$$
\begin{aligned}
& J\left(S_{u}, d \Sigma, \bar{S}_{v} ; Z\right) \\
& \quad=|\Sigma||F|^{2}\left|C^{2}-D^{2}\right|^{2} \\
& \quad=2^{n(1-m)} \prod_{i=1}^{n} \sigma_{i}^{2(m-n)+1} \prod_{1 \leq i<k \leq n}\left(\sigma_{i}^{2}-\sigma_{k}^{2}\right)^{2} .
\end{aligned}
$$

Now, from (48), (51), and (52) if follows that

$$
\begin{aligned}
& f_{U, \Sigma, V}(U, \Sigma, V) \\
& \quad=f_{\Delta}(\Sigma) 2^{n(1-m)} \prod_{i=1}^{n} \sigma_{i}^{2(m-n)+1} \prod_{1 \leq i<k \leq n}\left(\sigma_{i}^{2}-\sigma_{k}^{2}\right)^{2} .
\end{aligned}
$$

From (54), we immediately obtain that $U, \Sigma, V$ are statistically independent, and therefore (11) is proved. It also follows that $f_{U}$ and $f_{V}$ are constant over their respective domains, which proves (12) and (14). Finally, integrating (54) with respect to $d U$ and $d V$ we get the marginal density (13) as

\begin{tabular}{|c|c|c|c|c|c|c|c|c|}
\hline & $\begin{array}{l}\operatorname{Re}\left([Z]_{i i .}\right) \\
1 \leq i \leq n\end{array}$ & $\begin{array}{l}\operatorname{Im}\left([Z]_{i . i}\right) \\
1 \leq i \leq n\end{array}$ & $\begin{array}{l}\operatorname{Re}\left([Z]_{i k}\right) \\
1 \leq i<k \leq\end{array}$ & $\begin{array}{l}\operatorname{Re}\left([Z]_{i k}\right) \\
1 \leq k<i \leq n\end{array}$ & $\begin{array}{l}\operatorname{Im}\left([Z]_{i k}\right) \\
1 \leq i<k \leq n\end{array}$ & $\begin{array}{l}\operatorname{Im}\left([Z]_{i k}\right) \\
1 \leq k<i \leq n\end{array}$ & $\begin{array}{l}\operatorname{Re}\left([Z]_{i k}\right) \\
1 \leq i \leq n \\
1 \leq n<k \leq m\end{array}$ & $\begin{array}{l}\operatorname{Im}\left([Z]_{i k}\right) \\
1 \leq i \leq n \\
1 \leq n<k \leq m\end{array}$ \\
\hline $\begin{array}{l}\eta_{i} \\
\mu_{i i .}^{\circ} \\
1 \leq i \leq n\end{array}$ & $\begin{array}{l}I \\
0\end{array}$ & $\begin{array}{l}0 \\
\Sigma\end{array}$ & $\begin{array}{l}0 \\
0\end{array}$ & $\begin{array}{l}0 \\
0\end{array}$ & $\begin{array}{l}0 \\
0\end{array}$ & $\begin{array}{l}0 \\
0\end{array}$ & $\begin{array}{l}0 \\
0\end{array}$ & $\begin{array}{l}0 \\
0\end{array}$ \\
\hline $\begin{array}{l}\mu_{i k}^{\Re} \\
\nu_{i k}^{\Re} \\
1 \leq i<k \leq n\end{array}$ & $\begin{array}{l}0 \\
0\end{array}$ & $\begin{array}{c}0 \\
H^{\Re}\end{array}$ & $\begin{array}{c}C \\
-D\end{array}$ & $\begin{array}{c}-D \\
C\end{array}$ & $\begin{array}{l}0 \\
0\end{array}$ & $\begin{array}{l}0 \\
0\end{array}$ & $\begin{array}{l}0 \\
0\end{array}$ & $\begin{array}{l}0 \\
0\end{array}$ \\
\hline $\begin{array}{l}\mu_{i k}^{\Im} \\
\nu_{i k}^{\Im} \\
1 \leq i<k \leq n\end{array}$ & $\begin{array}{l}0 \\
0\end{array}$ & $\begin{array}{c}0 \\
H^{\Im}\end{array}$ & $\begin{array}{l}0 \\
0\end{array}$ & $\begin{array}{l}0 \\
0\end{array}$ & $\begin{array}{c}C \\
-D\end{array}$ & $\begin{array}{c}D \\
-C\end{array}$ & $\begin{array}{l}0 \\
0\end{array}$ & $\begin{array}{l}0 \\
0\end{array}$ \\
\hline $\begin{array}{l}q_{i k}^{\Re,} \\
q_{i k}^{\Im} \\
1 \leq i<k \leq n\end{array}$ & $\begin{array}{l}0 \\
0\end{array}$ & $\begin{array}{l}0 \\
0\end{array}$ & $\begin{array}{l}0 \\
0\end{array}$ & $\begin{array}{l}0 \\
0\end{array}$ & $\begin{array}{l}0 \\
0\end{array}$ & $\begin{array}{l}0 \\
0\end{array}$ & $\begin{array}{c}F \\
0\end{array}$ & $\begin{array}{l}0 \\
F\end{array}$ \\
\hline
\end{tabular}

$$
\begin{aligned}
f_{\Sigma}(\Sigma) & =\int \cdots \int f_{U, \Sigma, V}(U, \Sigma, V) d U d V \\
& =\Upsilon_{\mathbb{C}} f_{\Delta}(\Sigma) \prod_{i=1}^{n} \sigma_{i}^{2(m-n)+1} \prod_{1 \leq i<k \leq n}\left(\sigma_{i}^{2}-\sigma_{k}^{2}\right)^{2}
\end{aligned}
$$

where $\Upsilon_{\mathbb{C}}$ is a constant computed as follows. Let $\mathcal{G}_{\mathcal{U}}(n)$ be the unitary group of order $n$, and let $\mathcal{C}_{m, n}$ be the complex manifold $\mathcal{C}_{m, n}=\left\{V \in \mathbb{C}^{m, n}: V^{*} V=I_{n} ;[V]_{1, i}>0, i=1, \ldots, n\right\}$. Then, the volumes of these two sets are given by (see for instance [23])

$$
\begin{aligned}
\operatorname{vol}\left(\mathcal{G}_{\mathcal{U}}(n)\right) & =(2 \pi)^{n(n+1) / 2} \prod_{k=1}^{n} \frac{1}{(n-k) !} \\
\operatorname{vol}\left(\mathcal{C}_{m, n}\right) & =\frac{(2 \pi)^{m n-n(n-1) / 2}}{(2 \pi)^{n}} \prod_{k=1}^{n} \frac{1}{(m-k) !} .
\end{aligned}
$$

Therefore, from (55)

$$
\begin{aligned}
\Upsilon_{\mathbb{C}} & =2^{n(1-m)} \operatorname{vol}\left(\mathcal{G}_{\mathcal{U}}(n)\right) \operatorname{vol}\left(\mathcal{C}_{m, n}\right) \\
& =\frac{2^{n} \pi^{m n}}{\prod_{k=1}^{n}(n-k) !(m-k) !}
\end{aligned}
$$

This concludes the proof of Theorem 1. 


\section{B. Proof of Theorem 2}

This proof follows the lines of the one given for Theorem 1. Indeed, the derivation up to the expression of $Z$ as

$$
Z=\left[S_{u} \Sigma-\Sigma S_{v}+d \Sigma \mid \Sigma Q\right]
$$

is identical to the complex case, considering that all the involved quantities are now real, and $S_{v}, S_{u}$ are skew-symmetric. In particular, we have again that

$$
J(U, \Sigma, V)=J\left(S_{u}, d \Sigma, \bar{S}_{v} ; Z\right) .
$$

We now examine the number of free variables that describe the quantities of interest. The matrix $\Delta$ is described by means of $n m$ real variables. The orthogonal matrix $U$ is described by means of $n_{u} \doteq(n / 2)(n-1)$ real variables, $\Sigma$ is described by means of its $n$ diagonal entries, therefore $V$ is described by the remaining $n_{v} \doteq n m-(n / 2)(n-1)-n=n(m-n)+(n / 2)(n-1)$ real variables. The differentials $d U, d V, d \Sigma$ are described by the same number of free variables as, respectively, $U, V$, and $\Sigma$. Therefore, $S_{u}$ and $\bar{S}_{v}^{T}=\left[S_{v}^{T} \mid Q\right]$ are described by $n_{u}$ and $n_{v}$ variables, respectively. Since $d \Sigma$ is diagonal, we choose its $n$ free variables as the diagonal entries $\eta_{i}=d \sigma_{i}, 1 \leq i \leq n$. Since $S_{u}$ is skew-symmetric, we choose the $n_{u}$ free variables $\mu_{i k}$, as the coefficients of the standard orthonormal basis of the space of $n \times n$ skew-symmetric matrices. Therefore, using the notation introduced in (53), Appendix A, we write

$$
S_{u}=\sum_{1 \leq i<k \leq n} \mu_{i k} B_{i k}^{\Re}
$$

Similarly, considering that the matrix $\bar{S}_{v}=\left[S_{v}^{T} \mid Q\right]^{T}$ is the first (block) column of the $(m \times m)$ skew-symmetric matrix $\bar{V}^{T} d \bar{V}$, we choose $n(n-1) / 2$ free variables $\nu_{i k}$ such that

$$
S_{v}=\sum_{1 \leq i<k \leq n} \nu_{i k} B_{i k}^{\Re}
$$

The remaining $n_{v}-n(n-1) / 2=n(m-n)$ free variables, $q_{i k}$, are needed to describe $Q$

$$
[Q]_{i k}=\frac{1}{\sqrt{2}} q_{i k}, \quad 1 \leq i \leq n, 1 \leq k \leq m-n .
$$

The $(i, k)$ element of $Z$ may now be expressed as

$$
[Z]_{i k}=\left\{\begin{array}{lc}
\mu_{i k} \sigma_{k}-\nu_{i k} \sigma_{i}, & 1 \leq i<k \leq n \\
-\mu_{k i} \sigma_{k}+\nu_{k i} \sigma_{i}, & 1 \leq k<i \leq n \\
\eta_{i}, & i=k, 1 \leq i \leq n \\
q_{i r} \sigma_{i}, & r=k-n ; 1 \leq i \leq n \\
& n<k \leq m
\end{array}\right.
$$

To compute the Jacobian $J\left(S_{u}, d \Sigma, \bar{S}_{v} ; Z\right)$, we construct the scheme of partial derivatives shown at the bottom of the page, where where

$$
\begin{aligned}
& C=\frac{1}{\sqrt{2}} \operatorname{blockdiag}\left(\operatorname{diag}\left(\sigma_{2}, \ldots, \sigma_{n}\right), \operatorname{diag}\left(\sigma_{3}, \ldots, \sigma_{n}\right), \ldots,\right. \\
& \left.\operatorname{diag}\left(\sigma_{n-1}, \sigma_{n}\right), \sigma_{n}\right) \\
& D=\frac{1}{\sqrt{2}} \operatorname{blockdiag}\left(\sigma_{1} I_{n-1}, \sigma_{2} I_{n-2}, \ldots, \sigma_{n-2} I_{2}, \sigma_{n-1}\right) \\
& F=\frac{1}{\sqrt{2}} \operatorname{blockdiag}\left(\sigma_{1} I_{m-n}, \sigma_{2} I_{m-n}, \ldots, \sigma_{n-1} I_{m-n}\right. \\
& \left.\sigma_{n} I_{m-n}\right)
\end{aligned}
$$

\begin{tabular}{|c|c|c|c|c|}
\hline & $\begin{array}{l}{[Z]_{i i}} \\
1 \leq i \leq n\end{array}$ & $\begin{array}{l}{[Z]_{i k}} \\
1 \leq i<k \leq n\end{array}$ & $\begin{array}{l}{[Z]_{i k}} \\
1 \leq k<i \leq n\end{array}$ & $\begin{array}{l}{[Z]_{i k}} \\
1 \leq i \leq n \\
1 \leq n<k \leq m\end{array}$ \\
\hline $\begin{array}{l}\eta_{i} ; \\
1 \leq i \leq n\end{array}$ & $I$ & 0 & 0 & 0 \\
\hline $\begin{array}{l}\mu_{i k} ; \\
1 \leq i<k \leq n\end{array}$ & 0 & $C$ & $-D$ & 0 \\
\hline $\begin{array}{l}\nu_{i k} \\
1 \leq i<k \leq n\end{array}$ & 0 & $-D$ & $C$ & 0 \\
\hline $\begin{array}{l}q_{i k} \\
1 \leq i \leq n \\
1 \leq n<k \leq m\end{array}$ & 0 & 0 & 0 & $F$ \\
\hline
\end{tabular}

The matrix of partial derivatives is block diagonal and therefore its determinant is given by

$$
J\left(S_{u}, d \Sigma, \bar{S}_{v} ; Z\right)=|\Sigma||F|\left|\begin{array}{rr}
C & -D \\
-D & C
\end{array}\right| .
$$

Using Schur complement, we have that

$$
\begin{aligned}
& J\left(S_{u}, d \Sigma, \bar{S}_{v} ; Z\right) \\
& \quad=|\Sigma||F|\left|C^{2}-D^{2}\right| \\
& \quad=2^{n(1-m) / 2} \prod_{i=1}^{n} \sigma_{i}^{m-n} \prod_{1 \leq i<k \leq n}\left(\sigma_{i}^{2}-\sigma_{k}^{2}\right) .
\end{aligned}
$$

Now, from (56) if follows that

$$
\begin{aligned}
& f_{U, \Sigma, V}(U, \Sigma, V) \\
& \quad=f_{\Delta}(\Sigma) 2^{n(1-m) / 2} \prod_{i=1}^{n} \sigma_{i}^{m-n} \prod_{1 \leq i<k \leq n}\left(\sigma_{i}^{2}-\sigma_{k}^{2}\right) .
\end{aligned}
$$

From (57) we immediately obtain that $U, \Sigma, V$ are statistically independent, and therefore (16) is proved. It also follows that $f_{U}$ 
and $f_{V}$ are constant over their respective domains, which proves (17) and (19). Finally, integrating (57) with respect to $d U$ and $d V$ we get the marginal density (18) as

$$
\begin{aligned}
f_{\Sigma}(\Sigma) & =\int \cdots \int f_{U, \Sigma, V}(U, \Sigma, V) d U d V \\
& =\Upsilon_{\mathbb{R}} f_{\Delta}(\Sigma) \prod_{i=1}^{n} \sigma_{i}^{m-n} \prod_{1 \leq i<k \leq n}\left(\sigma_{i}^{2}-\sigma_{k}^{2}\right)
\end{aligned}
$$

where $\Upsilon_{\mathbb{R}}$ is a constant computed as follows. Let $\mathcal{G}_{\mathcal{O}}(n)$ be the orthogonal group of order $n$, and let $\mathcal{R}_{m, n}$ be the real manifold $\mathcal{R}_{m, n}=\left\{V \in \mathbb{R}^{m, n}: V^{T} V=I_{n} ;[V]_{1, i}>0, i=1, \ldots, n\right\}$. Then, the volumes of the two previous sets are given by (see for instance [23])

$$
\begin{aligned}
\operatorname{vol}\left(\mathcal{G}_{\mathcal{O}}(n)\right) & =(8 \pi)^{n(n-1) / 4} \prod_{k=1}^{n} \frac{\Gamma\left(\frac{k-1}{2}\right)}{\Gamma(k-1)} \\
\operatorname{vol}\left(\mathcal{R}_{m, n}\right) & =\frac{(8 \pi)^{m n / 2-n(n+1) / 4}}{2^{n}} \prod_{k=m-n+1}^{m} \frac{\Gamma\left(\frac{k-1}{2}\right)}{\Gamma(k-1)}
\end{aligned}
$$

where, for continuity, we assume

$$
\left.\frac{\Gamma\left(\frac{k-1}{2}\right)}{\Gamma(k-1)}\right|_{k=1}=2
$$

From (58) it finally follows that

$$
\begin{aligned}
\Upsilon_{\mathbb{R}} & =2^{n(1-m) / 2} \operatorname{vol}\left(\mathcal{G}_{\mathcal{O}}(n)\right) \operatorname{vol}\left(\mathcal{R}_{m, n}\right) \\
& =\frac{(8 \pi)^{n(m-1) / 2}}{2^{n(m+1) / 2}} \prod_{k=1}^{n} \frac{\Gamma\left(\frac{k-1}{2}\right)}{\Gamma(k-1)} \prod_{k=m-n+1}^{m} \frac{\Gamma\left(\frac{k-1}{2}\right)}{\Gamma(k-1)} .
\end{aligned}
$$

This concludes the proof of Theorem 2 .

\section{Proof of Theorem 3}

Let us rewrite (27) in the form

$$
f_{x}(x)=K_{x}\left|\mathcal{V}_{n}^{T} \mathcal{V}_{n}\right| \mu_{n}(x)
$$

where, for $k=1, \ldots, n$

$$
\mu_{k}(x) \doteq \prod_{i=1}^{k} x_{i}^{m-n} .
$$

For any nonsingular matrix $A, M \doteq A^{T} A$ is symmetric and

$$
f_{x}(x)=\frac{K_{x}}{|M|}\left|\mathcal{V}_{n}^{T} M \mathcal{V}_{n}\right| \mu_{n}(x) .
$$

Define further

$$
Z_{k} \doteq \mathcal{V}_{k}^{T} M \mathcal{V}_{k}, \quad \text { for } k=1, \ldots, n .
$$

Let now $A$ be given by

$$
A \doteq\left[\begin{array}{ccccc}
a_{0}^{(0)} & a_{1}^{(0)} & a_{2}^{(0)} & \cdots & a_{n-1}^{(0)} \\
a_{0}^{(1)} & a_{1}^{(1)} & a_{2}^{(1)} & \cdots & a_{n-1}^{(1)} \\
\vdots & \vdots & \vdots & & \vdots \\
a_{0}^{(n-1)} & a_{1}^{(n-1)} & a_{2}^{(n-1)} & \cdots & a_{n-1}^{(n-1)}
\end{array}\right]
$$

then

$$
A \mathcal{X}_{i}=\left[\begin{array}{c}
L_{0}\left(x_{i}\right) \\
L_{1}\left(x_{i}\right) \\
L_{2}\left(x_{i}\right) \\
\vdots \\
L_{n-1}\left(x_{i}\right)
\end{array}\right]
$$

where $L_{k}\left(x_{i}\right)$ are polynomials of degree $n-1$

$$
\begin{gathered}
L_{k}\left(x_{i}\right)=a_{0}^{(k)}+a_{1}^{(k)} x_{i}+a_{2}^{(k)} x_{i}^{2}+\cdots+a_{n-1}^{(k)} x_{i}^{n-1}, \\
k=0, \ldots, n-1 .
\end{gathered}
$$

We choose $A$ such that the polynomials $L_{k}\left(x_{i}\right), k=0, \ldots, n-$ 1 form an orthogonal polynomial basis on the interval $x_{i} \in$ $[0,1]$, with respect to the weight function $x_{i}^{m-n}$. That is,

$$
\int_{0}^{1} L_{k}\left(x_{i}\right) L_{h}\left(x_{i}\right) x_{i}^{m-n} d x_{i}= \begin{cases}1, & \text { if } k=h \\ 0, & \text { otherwise }\end{cases}
$$

for $k, h=0, \ldots, n-1$. This condition may be written in matrix form as

$$
A\left(\int_{0}^{1} \mathcal{X}_{i} \mathcal{X}_{i}^{T} x_{i}^{m-n} d x_{i}\right) A^{T}=I
$$

and the integral term is easily evaluated as

$$
\begin{aligned}
{[R]_{r, \ell} } & \doteq\left[\int_{0}^{1} \mathcal{X}_{i} \mathcal{X}_{i}^{T} x_{i}^{m-n} d x_{i}\right]_{r, \ell} \\
& =\frac{1}{r+\ell+m-n-1}, \quad r, \ell=1, \ldots, n .
\end{aligned}
$$

Taking the eigenvalue decomposition of the symmetric matrix $R=P \Lambda P^{T}$ with $P$ orthogonal, it follows immediately that the orthogonality condition (62) is satisfied for

$$
A=\Lambda^{-1 / 2} P^{T}
$$

therefore $M=A^{T} A=P \Lambda^{-1} P^{T}=R^{-1}$.

It is now straightforward to show that the matrix $Z_{k}$ satisfies the conditions of the Dyson-Mehta Theorem for the integrals of certain determinants; see Appendix E. In particular, we have that

$$
\left[Z_{k}\right]_{i, j}=\sum_{k=0}^{n-1} L_{k}\left(x_{i}\right) L_{k}\left(x_{j}\right)=\psi\left(x_{i}, x_{j}\right)
$$

and

$$
\int_{0}^{1} \psi\left(x_{i}, x_{i}\right) x_{i}^{m-n} d x_{i}=n .
$$

Therefore, from Theorem 4 in Appendix E we obtain ${ }^{1}$

$$
\begin{aligned}
& \int_{0}^{1}\left|Z_{k}\left(x_{1}, \ldots, x_{k}\right)\right| x_{k}^{m-n} d x_{k} \\
& \quad=(n-k+1)\left|Z_{k-1}\left(x_{1}, \ldots, x_{k-1}\right)\right| .
\end{aligned}
$$


Applying (63) recursively for $k$ going backward from $n$ to $i+1$, we have that

$$
\begin{aligned}
& \int_{0}^{1} \ldots \int_{0}^{1}\left|Z_{n}\left(x_{1}, \ldots, x_{n}\right)\right|\left(x_{i+1} \ldots x_{n}\right)^{m-n} d x_{i+1} \ldots d x_{n} \\
& \quad=(n-i) !\left|Z_{i}\left(x_{1}, \ldots, x_{i}\right)\right| .
\end{aligned}
$$

By means of (60) we obtain the marginal density

$$
f_{i}\left(x_{1}, \ldots, x_{i}\right)=K_{x} \frac{(n-i) !}{|M|} \mu_{i}(x)\left|Z_{i}\left(x_{1}, \ldots, x_{i}\right)\right| .
$$

The proof is then completed substituting (59) and (61) in the above expression.

\section{Proof of Corollary 1}

Let $Z_{i} \doteq \mathcal{V}_{i}^{T} M \mathcal{V}_{i}$, and recall that $\mathcal{V}_{i}=\left[\mathcal{V}_{i-1} \mathcal{X}_{i}\right]$, then

$$
\left|Z_{i}\right|=\left|\begin{array}{cc}
Z_{i-1} & \mathcal{V}_{i-1}^{T} M \mathcal{X}_{i} \\
\mathcal{X}_{i}^{T} M \mathcal{V}_{i-1} & \mathcal{X}_{i}^{T} M \mathcal{X}_{i}
\end{array}\right|
$$

Using the Schur rule for the above determinant we get

$$
\begin{aligned}
\left|Z_{i}\left(x_{1}, \ldots, x_{i}\right)\right|= & \left|Z_{i-1}\left(x_{1}, \ldots, x_{i-1}\right)\right| \\
& \cdot \mathcal{X}_{i}^{T} W_{i-1} \mathcal{X}_{i} ; \quad i=2, \ldots, n \\
\left|Z_{1}\left(x_{1}\right)\right|= & \mathcal{X}_{1}^{T} M \mathcal{X}_{1}
\end{aligned}
$$

where the matrix

$$
W_{i-1}=M-M \mathcal{V}_{i-1} Z_{i-1}^{-1} \mathcal{V}_{i-1}^{T} M
$$

contains the variables up to $i-1$. The term $\mathcal{X}_{i}^{T} W_{i-1} \mathcal{X}_{i}$ can be written as a polynomial in the variable $x_{i}$, with coefficients depending on $x_{1}, \ldots, x_{i-1}$. It is straightforward to verify that these coefficients are given by the sum of the elements of the anti-diagonals of $W_{i-1}$, that is

$$
\mathcal{X}_{i}^{T} W_{i-1} \mathcal{X}_{i}=\sum_{k=0}^{2(n-1)} b_{k} x_{i}^{k}
$$

where

$$
b_{k}=\sum_{\{r+\ell=k+2\}}\left[W_{i-1}\right]_{r, \ell}, \quad k=0, \ldots, 2(n-1) .
$$

Combining the expressions (64)-(66) we therefore prove the statements (32) and (37).

\section{E. On the Integral of Certain Determinants}

In this Appendix we report a result on the computation of the integral of a determinant. The proof of this theorem can be found in [27].

Theorem 4 (Dyson-Mehta): Let $Z_{n} \in \mathbb{R}^{n, n}$ be a $n \times n$ symmetric matrix such that

1) $[Z]_{i, j}=\psi\left(x_{i}, x_{j}\right)$, i.e., $[Z]_{i, j}$ depends only on $x_{i}$ and $x_{j}$;

2) $\int \psi(x, x) d \mu(x)=c$;

3) $\int \psi(x, y) \psi(y, z) d \mu(y)=\psi(x, z)$;

where $d \mu$ is a suitable measure and $c$ is a constant. Then

$$
\int \operatorname{det}\left(Z_{n}\right) d \mu\left(x_{n}\right)=(c-n+1) \operatorname{det}\left(Z_{n-1}\right)
$$

where $Z_{n-1}$ is the $(n-1) \times(n-1)$ matrix obtained from $Z_{n}$ by removing the row and the column containing $x_{n}$.

\section{F. Transformations of Random Variables}

Let $X$ and $Y$ be two random matrices with the same number $p$ of free elements $x_{1}, \ldots, x_{p}$ and $y_{1}, \ldots, y_{p}$, respectively. Let the p.d.f. of $Y$ be $f_{Y}(Y)$, and let $X, Y$ be related by a one-to-one transformation $Y=g(X)$. Then, [1] the p.d.f. $f_{X}$ is

$$
f_{X}(X)=f_{Y}(g(X)) J(X ; Y)
$$

where the Jacobian $J(X ; Y)$ is defined as

$$
J(X ; Y) \doteq\left|\begin{array}{cccc}
\frac{\partial y_{1}}{\partial x_{1}} & \frac{\partial y_{2}}{\partial x_{1}} & \cdots & \frac{\partial y_{p}}{\partial x_{1}} \\
\frac{\partial y_{1}}{\partial x_{2}} & \frac{\partial y_{2}}{\partial x_{2}} & \cdots & \frac{\partial y_{p}}{\partial x_{2}} \\
\vdots & \vdots & & \vdots \\
\frac{\partial y_{1}}{\partial x_{p}} & \frac{\partial y_{2}}{\partial x_{p}} & \cdots & \frac{\partial y_{p}}{\partial x_{p}}
\end{array}\right|
$$

More generally, if $X_{1}, \ldots, X_{k}$ and $Y_{1}, \ldots, Y_{m}$ satisfy the equations $Y_{i}=g_{i}\left(x_{1}, \ldots, x_{k}\right), i=1, \ldots, m$, and $\left(X_{1}, \ldots, X_{k},\right),\left(Y_{1}, \ldots, Y_{m}\right)$ have the free elements $x_{1}, \ldots, x_{p}$ and $y_{1}, \ldots, y_{p}$, respectively, then the Jacobian of the transformation from $\left(X_{1}, \ldots, X_{k}\right)$ to $\left(Y_{1}, \ldots, Y_{m}\right)$ will be denoted as $J\left(X_{1}, \ldots, X_{k} ; Y_{1}, \ldots, Y_{m}\right)$.

\section{G. General Properties of Jacobians}

For completeness, we state here some basic properties of Jacobians that are used in the proofs of Theorems 1 and 2. The proofs that are not reported here can be found in [16] and [29].

G.1: Chain rule for Jacobians

$$
J(X ; Y)=J(X ; Z) J(Z ; Y) .
$$

G.2: Given a matrix transformation (linear or not) $Y=F(X)$, then the transformation of the differentials, $d Y=d F(X)$ is linear, and

$$
J(X ; Y)=J(d X ; d Y) .
$$

G.3: The Jacobian of the transformation

$$
Y=A X
$$

where $X \in \mathbb{R}^{n, m}, A \in \mathbb{R}^{n, n}$, is given by

$$
J(X ; Y)=|A|^{m} .
$$

Similarly, the Jacobian of the transformation $Y=X B$, with $B \in \mathbb{R}^{m, m}$, is given by

$$
J(X ; Y)=|B|^{n} .
$$

G.4: The Jacobian of the transformation

$$
Y=A X B
$$

where $X \in \mathbb{R}^{n, m}, A \in \mathbb{R}^{n, n}, B \in \mathbb{R}^{m, m}$, is given by

$$
J(X ; Y)=|A|^{m}|B|^{n} .
$$

Remark that if $A$ and $B$ are orthogonal, then $J(X ; Y)=1$. 
G.5: The Jacobian of the transformation

$$
Y=A X B
$$

where $X \in \mathbb{C}^{n, m}, A \in \mathbb{C}^{n, n}, B \in \mathbb{C}^{m, m}$ is given by

$$
J(X ; Y)=|\tilde{A}|^{2 m}|\tilde{B}|^{2 n}
$$

where

$$
\tilde{A}=\left[\begin{array}{cc}
\operatorname{Re}(A) & -\operatorname{Im}(A) \\
\operatorname{Im}(A) & \operatorname{Re}(A)
\end{array}\right], \quad \tilde{B}=\left[\begin{array}{cc}
\operatorname{Re}(B) & -\operatorname{Im}(B) \\
\operatorname{Im}(B) & \operatorname{Re}(B)
\end{array}\right] .
$$

Proof: To prove this, first note that, by G.1, $J(X ; Y)=$ $J(X ; Z) J(Z ; Y)$, where $Z=A X$. Write the linear equation $Z=A X$ in terms of the real and imaginary parts of the terms

$$
\left[\begin{array}{l}
\operatorname{Re}(Z) \\
\operatorname{Im}(Z)
\end{array}\right]=\tilde{A}\left[\begin{array}{l}
\operatorname{Re}(X) \\
\operatorname{Im}(X)
\end{array}\right]
$$

then, by G.3, $J(X ; Z)=|\tilde{A}|^{2 m}$. By a similar reasoning $J(Z ; Y)=|\tilde{B}|^{2 n}$.

Notice that, if $A$ is unitary, then it can be easily seen that $\tilde{A}$ is orthogonal. Therefore, for $A, B$ unitary, $J(X ; Y)=1$.

\section{H. Plant Data for the Numerical Example}

See equations (A), (B), and (C).

$$
\begin{aligned}
& A=\left[\begin{array}{cccccccccccccc}
0 & 0 & 0 & 0 & 0 & 1 & 0 & 0 & 0 & 0 & 0 & 0 & 0 & 0 \\
0 & 0 & 0 & 0 & 0 & 0 & 1 & 0 & 0 & 0 & 0 & 0 & 0 & 0 \\
0 & 0 & 0 & 0 & 0 & 0 & 0 & 1 & 0 & 0 & 0 & 0 & 0 & 0 \\
0 & 0 & 0 & 0 & 0 & 0 & 0 & 0 & 1 & 0 & 0 & 0 & 0 & 0 \\
0 & 0 & 0 & 0 & 0 & 0 & 0 & 0 & 0 & 1 & 0 & 0 & 0 & 0 \\
-200 & 100 & 0 & 0 & 0 & -2 & 1 & 0 & 0 & 0 & 27.3 & -100 & 26.8 & -106 \\
100 & -200 & 100 & 0 & 0 & 1 & -2 & 1 & 0 & 0 & -99.8 & -173 & -101 & 0.794 \\
0 & 100 & -200 & 100 & 0 & 0 & 1 & -2 & 1 & 0 & 20.5 & -101 & 91.5 & -85.2 \\
0 & 0 & 100 & -200 & 100 & 0 & 0 & 1 & -2 & 1 & -58.4 & 0.870 & -92.9 & 105 \\
0 & 0 & 0 & 100 & -200 & 0 & 0 & 0 & 1 & -2 & 0 & 0 & 0 & 0 \\
0 & 3820 & 1190 & 25 & -292 & 0 & 0 & 0 & 0 & 0 & 0 & -3820 & -1190 & -25 \\
0 & 210 & 20.2 & 1.85 & -6.44 & 0 & 0 & 0 & 0 & 0 & 0 & -210 & -20.2 & -1.85 \\
0 & 2.26 & 126 & 4.19 & -5.68 & 0 & 0 & 0 & 0 & 0 & 0 & -2.26 & -126 & -4.19 \\
0 & -0.211 & 4.17 & 120 & 0.708 & 0 & 0 & 0 & 0 & 0 & 0 & 0.211 & -4.17 & -120 \\
0 & 0.121 & -6.34 & 0.899 & 117 & 0 & 0 & 0 & 0 & 0 & 0 & -0.121 & 6.34 & -0.899 \\
0 & 15500 & 12900 & -484 & -2210 & 0 & 0 & 0 & 0 & 0 & -173 & -15500 & -12900 & 378 \\
0 & 14600 & 3150 & 183 & -862 & 0 & 0 & 0 & 0 & 0 & 0.192 & -15000 & -3150 & -182 \\
0 & 399 & 3460 & 399 & -342 & 0 & 0 & 0 & 0 & 0 & 20.5 & -400 & -3570 & -384 \\
0 & -11.8 & 387 & 3220 & 203 & 0 & 0 & 0 & 0 & 0 & -58.4 & 12.7 & -380 & -3320 \\
0 & 7.75 & -370 & 223 & 2990 & 0 & 0 & 0 & 0 & 0 & 0 & -7.75 & 370 & -123 \\
0 & -1.39 & 0 & 0 & 0 & 0 & 0 & 0 & 0 & 0 & 0 & 0 & 0 & 0 \\
0 & 0 & -1.39 & 0 & 0 & 0 & 0 & 0 & 0 & 0 & 0 & 0 & 0 & 0 \\
0 & 0 & 0 & -1.39 & 0 & 0 & 0 & 0 & 0 & 0 & 0 & 0 & 0 & 0 \\
0 & 0 & 0 & 0 & -1.39 & 0 & 0 & 0 & 0 & 0 & 0 & 0 & 0 & 0
\end{array}\right. \\
& \left.\begin{array}{cccccccccc}
0 & 0 & 0 & 0 & 0 & 0 & 0 & 0 & 0 & 0 \\
0 & 0 & 0 & 0 & 0 & 0 & 0 & 0 & 0 & 0 \\
0 & 0 & 0 & 0 & 0 & 0 & 0 & 0 & 0 & 0 \\
0 & 0 & 0 & 0 & 0 & 0 & 0 & 0 & 0 & 0 \\
0 & 0 & 0 & 0 & 0 & 0 & 0 & 0 & 0 & 0 \\
15.8 & -5.14 & -0.931 & -2.43 & 14.5 & 1.64 & 0 & 0 & 0 & 0 \\
-0.171 & -1.11 & -1.36 & -1.17 & 0.120 & -0.00829 & 0 & 0 & 0 & 0 \\
12.6 & 3.34 & -0.973 & 1.61 & -5.78 & -0.798 & 0 & 0 & 0 & 0 \\
-64.5 & -5.03 & 0.00790 & -1.04 & 5.89 & -0.0457 & 0 & 0 & 0 & 0 \\
0 & 0 & 0 & 0 & 0 & 0 & 0 & 0 & 0 & 0 \\
292 & 1 & 0 & 0 & 0 & 0 & 0 & 0 & 0 & 0 \\
6.44 & 0 & 1 & 0 & 0 & 0 & 0 & 0 & 0 & 0 \\
5.68 & 0 & 0 & 1 & 0 & 0 & 0 & 0 & 0 & 0 \\
-0.708 & 0 & 0 & 0 & 1 & 0 & 0 & 0 & 0 & 0 \\
-117 & 0 & 0 & 0 & 0 & 1 & 0 & 0 & 0 & 0 \\
2230 & -7.14 & 0.0692 & -2.43 & 14.5 & 1.64 & 0 & 0 & 0 & 0 \\
862 & -0.109 & -3.36 & -0.172 & 0.120 & -0.00829 & 0 & 0 & 0 & 0 \\
355 & 3.34 & 0.0267 & -0.393 & -4.78 & -0.798 & 0 & 0 & 0 & 0 \\
-167 & -5.03 & 0.00790 & -0.0419 & 3.89 & 0.954 & 0 & 0 & 0 & 0 \\
-3190 & 0 & 0 & 0 & 1 & -2 & 0 & 0 & 0 & 0 \\
0 & 0 & 0 & 0 & 0 & 0 & -48.3 & 0 & 0 & 0 \\
0 & 0 & 0 & 0 & 0 & 0 & 0 & -48.3 & 0 & 0 \\
0 & 0 & 0 & 0 & 0 & 0 & 0 & 0 & -48.3 & 0 \\
0 & 0 & 0 & 0 & 0 & 0 & 0 & 0 & 0 & -48.3
\end{array}\right]
\end{aligned}
$$




$$
B^{T}=\left[\begin{array}{cccccccccccc}
0 & 0 & 0 & 0 & 0 & -0.394 & 0.683 & -0.789 & 0.683 & -0.394 & 0 & 0 \\
0 & 0 & 0 & 0 & 0 & 0.612 & -0.612 & 0 & 0.612 & -0.612 & 0 & 0 \\
0 & 0 & 0 & 0 & 0 & -0.577 & 0 & 0.577 & 0 & -0.577 & 0 & 0 \\
0 & 0 & 0 & 0 & 0 & 0.354 & 0.354 & 0 & -0.354 & -0.354 & 0 & 0 \\
0 & 0 & 0 & 0 & 0 & -0.106 & -0.183 & -0.211 & -0.183 & -0.106 & 0 & 0 \\
0 & 0 & 0 & 0 & 0 & -0.394 & 0.683 & -0.789 & 0.683 & -0.394 & 0 & 0 \\
0 & 0 & 0 & 0 & 0 & 0.612 & -0.612 & 0 & 0.612 & -0.612 & 0 & 0 \\
0 & 0 & 0 & 0 & 0 & -0.577 & 0 & 0.577 & 0 & -0.577 & 0 & 0 \\
0 & 0 & 0 & 0 & 0 & 0.354 & 0.354 & 0 & -0.354 & -0.354 & 0 & 0 \\
0 & 0 & 0 & 0 & 0 & -0.106 & -0.183 & -0.211 & -0.183 & -0.106 & 0 & 0 \\
0 & 0 & 0 & 0 & 0 & 0 & 0 & 0 & 0 & 0 & 3820 & 210 \\
0 & 0 & 0 & 0 & 0 & 0 & 0 & 0 & 0 & 0 & 1190 & 20.2 \\
0 & 0 & 0 & 0 & 0 & 0 & 0 & 0 & 0 & 0 & 25 & 1.85 \\
0 & 0 & 0 & 0 & 0 & 0 & 0 & 0 & 0 & 0 & -292 & -6.44
\end{array}\right.
$$

$\left.\begin{array}{cccccccccccc}0 & 0 & 0 & 0 & 0 & 0 & 0 & 0 & 0 & 0 & 0 & 0 \\ 0 & 0 & 0 & 0 & 0 & 0 & 0 & 0 & 0 & 0 & 0 & 0 \\ 0 & 0 & 0 & 0 & 0 & 0 & 0 & 0 & 0 & 0 & 0 & 0 \\ 0 & 0 & 0 & 0 & 0 & 0 & 0 & 0 & 0 & 0 & 0 & 0 \\ 0 & 0 & 0 & 0 & 0 & 0 & 0 & 0 & 0 & 0 & 0 & 0 \\ 0 & 0 & 0 & 0 & 0 & 0 & 0 & 0 & 0 & 0 & 0 & 0 \\ 0 & 0 & 0 & 0 & 0 & 0 & 0 & 0 & 0 & 0 & 0 & 0 \\ 0 & 0 & 0 & 0 & 0 & 0 & 0 & 0 & 0 & 0 & 0 & 0 \\ 0 & 0 & 0 & 0 & 0 & 0 & 0 & 0 & 0 & 0 & 0 & 0 \\ 0 & 0 & 0 & 0 & 0 & 0 & 0 & 0 & 0 & 0 & 0 & 0 \\ 2.26 & -0.211 & 0.121 & 15500 & 14600 & 399 & -11.8 & 7.75 & 0 & 0 & 0 & 0 \\ 126 & 4.17 & -6.34 & 12900 & 3150 & 3460 & 387 & -370 & 0 & 0 & 0 & 0 \\ 4.19 & 120 & 0.899 & -484 & 183 & 399 & 3220 & 223 & 0 & 0 & 0 & 0 \\ -5.68 & 0.708 & 117 & -2210 & -862 & -342 & 203 & 2990 & 0 & 0 & 0 & 0\end{array}\right]$

$$
C=\left[\begin{array}{cccccccccccc}
0 & 0 & 0 & 0 & 0 & 0.394 & -0.683 & 0.789 & -0.683 & 0.394 & 0 & 0 \\
0 & 0 & 0 & 0 & 0 & -0.612 & 0.612 & 0 & -0.612 & 0.612 & 0 & 0 \\
0 & 0 & 0 & 0 & 0 & 0.577 & 0 & -0.577 & 0 & 0.577 & 0 & 0 \\
0 & 0 & 0 & 0 & 0 & -0.354 & -0.354 & 0 & 0.354 & 0.354 & 0 & 0 \\
0 & 0 & 0 & 0 & 0 & 0.106 & 0.183 & 0.211 & 0.183 & 0.106 & 0 & 0 \\
0.394 & -0.683 & 0.789 & -0.683 & 0.394 & 0 & 0 & 0 & 0 & 0 & 0 & 0 \\
-0.612 & 0.612 & 0 & -0.612 & 0.612 & 0 & 0 & 0 & 0 & 0 & 0 & 0 \\
0.577 & 0 & -0.577 & 0 & 0.577 & 0 & 0 & 0 & 0 & 0 & 0 & 0 \\
-0.354 & -0.354 & 0 & 0.354 & 0.354 & 0 & 0 & 0 & 0 & 0 & 0 & 0 \\
0.106 & 0.183 & 0.211 & 0.183 & 0.106 & 0 & 0 & 0 & 0 & 0 & 0 & 0 \\
0 & 0.0500 & 0 & 0 & 0 & 0 & 0 & 0 & 0 & 0 & 0 & 0 \\
0 & 0 & 0.0500 & 0 & 0 & 0 & 0 & 0 & 0 & 0 & 0 & 0 \\
0 & 0 & 0 & 0.0500 & 0 & 0 & 0 & 0 & 0 & 0 & 0 & 0 \\
0 & 0 & 0 & 0 & 0.0500 & 0 & 0 & 0 & 0 & 0 & 0 & 0
\end{array}\right.
$$

$\left.\begin{array}{llllllllllll}0 & 0 & 0 & 0 & 0 & 0 & 0 & 0 & 0 & 0 & 0 & 0 \\ 0 & 0 & 0 & 0 & 0 & 0 & 0 & 0 & 0 & 0 & 0 & 0 \\ 0 & 0 & 0 & 0 & 0 & 0 & 0 & 0 & 0 & 0 & 0 & 0 \\ 0 & 0 & 0 & 0 & 0 & 0 & 0 & 0 & 0 & 0 & 0 & 0 \\ 0 & 0 & 0 & 0 & 0 & 0 & 0 & 0 & 0 & 0 & 0 & 0 \\ 0 & 0 & 0 & 0 & 0 & 0 & 0 & 0 & 0 & 0 & 0 & 0 \\ 0 & 0 & 0 & 0 & 0 & 0 & 0 & 0 & 0 & 0 & 0 & 0 \\ 0 & 0 & 0 & 0 & 0 & 0 & 0 & 0 & 0 & 0 & 0 & 0 \\ 0 & 0 & 0 & 0 & 0 & 0 & 0 & 0 & 0 & 0 & 0 & 0 \\ 0 & 0 & 0 & 0 & 0 & 0 & 0 & 0 & 0 & 0 & 0 & 0 \\ 0 & 0 & 0 & 0 & 0 & 0 & 0 & 0 & 1.39 & 0 & 0 & 0 \\ 0 & 0 & 0 & 0 & 0 & 0 & 0 & 0 & 0 & 1.39 & 0 & 0 \\ 0 & 0 & 0 & 0 & 0 & 0 & 0 & 0 & 0 & 0 & 1.39 & 0 \\ 0 & 0 & 0 & 0 & 0 & 0 & 0 & 0 & 0 & 0 & 0 & 1.39\end{array}\right]$

\section{ACKNOWLEDGMENT}

The authors express gratitude to Prof. F. Ricci for his helpful comments on this line of research and, in particular, for his excellent technical suggestions regarding the proofs of Theorems
1 and 2. The detailed comments of Prof. F. Callier are also gratefully acknowledged.

\section{REFERENCES}

[1] T. W. Anderson, An Introduction to Multivariate Statistical Analysis. New York: Wiley, 1984. 
[2] E. W. Bai, R. Tempo, and M. Fu, "Worst case properties of the uniform distribution and randomized algorithms for robustness analysis," Math. Contr., Signals, Syst., vol. 11, pp. 183-196, 1998.

[3] B. R. Barmish and C. M. Lagoa, "The uniform distribution: A rigorous justification for its use in robustness analysis," Math. Contr., Signals, Syst., vol. 10, pp. 203-222, 1997.

[4] B. R. Barmish, C. M. Lagoa, and R. Tempo, "Radially truncated uniform distribution for probabilistic robustness of control systems," in Proc. American Control Conf., Albuquerque, NM, 1997.

[5] V. Blondel and J. N. Tsitsiklis, "A survey of computational complexity results in systems and control," Automatica, vol. 36, 2000.

[6] S. P. Boyd, L. El Ghaoui, E. Feron, and V. Balakrishnan, Linear Matrix Inequalities in Systems and Control Theory. Philadelphia, PA: SIAM, 1994.

[7] R. P. Braatz, P. M. Young, J. C. Doyle, and M. Morari, "Computational complexity of $\mu$ calculation," IEEE Trans. Automat. Contr., vol. 39, pp. $1000-1002,1994$

[8] G. Calafiore, "Structural properties of zeros for matrix second order systems: Theory and applications," Ph.D. dissertation (in Italian), Politecnico di Torino, Torino, 1997.

[9] G. Calafiore, F. Dabbene, and R. Tempo, "Uniform sample generation in $\ell_{p}$ balls for probabilistic robustness analysis," in Proc. IEEE Conf. Decision Control, Tampa, FL, 1998.

[10] — "Radial and uniform distributions in vector and matrix spaces for probabilistic robustness," in Topics in Control and Its Applications, D. Miller and L. Qiu, Eds. London: Springer-Verlag, 1999, pp. 17-31.

[11] — "The probabilistic real stability radius," in Proc. 14th World Congr. IFAC, Beijing, China, 1999

[12] G. Calafiore and F. Dabbene, "Polynomial-time random generation of uniform real matrices in the spectral norm ball," in Proc. IFAC Symp. Robust Control Design, Prague, Czech Republic, 2000.

[13] X. Chen and K. Zhou, "Order statistics and probabilistic robust control," Syst. Contr. Lett., vol. 35, pp. 175-182, 1998.

[14] H. Chernoff, "A measure of asymptotic efficiency for test of hypothesis based on the sum of observations," Annals Math. Statistics, vol. 23, pp. 493-507, 1952

[15] G. E. Coxson and C. L. DeMarco, "The computational complexity of approximating the minimal perturbation scaling to achieve instability in an interval matrix," Math. Contr., Signal Syst., vol. 7, pp. 279-291, 1994.

[16] W. L. Deemer and I. Olkin, "The Jacobians of certain matrix transformations useful in multivariate analysis," Biometrika, vol. 38, pp. 345-367, 1951.

[17] L. Devroye, Non-Uniform Random Variate Generation. New York: Springer-Verlag, 1986.

[18] _ "Random variate generation for multivariate unimodal densities," ACM Trans. Modeling Computer Simulation, vol. 7, pp. 447-477, 1997.

[19] A. El Bouhtouri and A. J. Pritchard, "Stability radii of linear systems with respect to stochastic perturbations," Syst. Contr. Lett., vol. 19, pp. 29-33, 1992.

[20] V. L. Girko, Theory of Random Determinants. Boston, MA: Kluwer, 1990.

[21] D. Hinrichsen and A. J. Pritchard, "Stability radii of linear systems," Syst. Contr. Lett., vol. 7, pp. 1-10, 1986.

[22] R. A. Horn and C. R. Johnson, Matrix Analysis. Cambridge, MA: Cambridge Univ. Press, 1988.

[23] L. K. Hua, Harmonic Analysis of Functions of Several Complex Variables in the Classical Domains. Providence, RI: American Mathematical Soc., 1979.

[24] P. P. Khargonekar and A. Tikku, "Randomized algorithms for robust control analysis have polynomial time complexity," in Proc. IEEE Conf. Decision Control, Kobe, Japan, 1996

[25] V. Koltchinskii, C. T. Abdallah, M. Ariola, P. Dorato, and D. Panchenko, "Improved sample complexity estimates for statistical learning control of uncertain systems," IEEE Trans. Automat. Contr., vol. 46, 2001. to appear.

[26] L. Lovasz and M. Simonovits, "Random walks in a convex body and an improved volume algorithm," Random Structures Algorithms, vol. 4, pp. 359-412, 1993

[27] M. L. Mehta, Random Matrices. . Boston, MA: Academic, 1991.

[28] A. Nemirovskii, "Several NP-hard problems arising in robust stability analysis," Math. Contr., Signals Syst., vol. 6, pp. 99-105, 1993.

[29] I. Olkin, "Note on the Jacobians of certain matrix transformations useful in multivariate analysis," Biometrika, vol. 40, pp. 43-46, 1953.

[30] S. Polijak and J. Rohn, "Checking robust nonsingularity is NP-hard," Math. Contr., Signals Syst., vol. 6, pp. 1-9, 1993.
[31] B. T. Polyak and P. S. Shcherbakov, "Random spherical uncertainty in estimation and robustness," IEEE Trans. Automat. Contr., vol. 46, 2001. to appear.

[32] L. Qiu, L. B. Bernhardsson, A. Rantzer, E. J. Davison, P. M. Young, and J. Doyle, "A formula for computation of the real stability radius," Automatica, vol. 6, pp. 879-890, 1995 .

[33] L. R. Ray and R. F. Stengel, "A Monte Carlo approach to the analysis of control system robustness," Automatica, vol. 29, pp. 229-236, 1993

[34] R. Y. Rubinstein, Simulation and the Monte Carlo Method. New York: Wiley, 1981.

[35] A. Selberg, "Bemerkninger om et multiplet integral," Norsk Matematisk Tidsskrift, vol. 26, pp. 71-78, 1944.

[36] G. W. Stewart, "The efficient generation of random orthogonal matrices with an application to condition estimators," SIAM J. Numerical Anal., vol. 17, no. 3, pp. 403-409, 1980.

[37] R. Tempo, E. W. Bai, and F. Dabbene, "Probabilistic robustness analysis: Explicit bounds for the minimum number of samples," Syst. Contr. Lett., vol. 30, pp. 237-242, 1997.

[38] R. Tempo and F. Dabbene, "Probabilistic robustness analysis and design of uncertain systems," in Dynamical Syst., Contr., Coding, Computer Vision, G. Picci and D. S. Gilliam, Eds: Birkhauser, 1999.

[39] _ - "Probabilistic methods for robustness analysis and design of uncertain systems," J. Soc. Instrument Control Engineers (SICE) Japan, 2000.

[40] M. Vidyasagar, A Theory of Learning and Generalization. London: Springer-Verlag, 1997.

[41] _ _ "Statistical learning theory: An introduction and applications to randomized algorithms," in Proc. European Control Conf., Brussels, Belgium, 1997.

[42] K. Zhou, J. C. Doyle, and K. Glover, Robust and Optimal Control. Upper Saddle River, NJ: Prentice-Hall, 1996.

[43] X. Zhu, Y. Huang, and J. Doyle, "Soft vs. hard bounds in probabilistic robustness analysis," in Proc. IEEE Conf. Decision Control, Kobe, Japan, 1996.

[44] K. Zyczkowski and M. Kus, "Random unitary matrices," J. Physics A, vol. 27 , no. 12 , pp. $4235-4245,1994$.

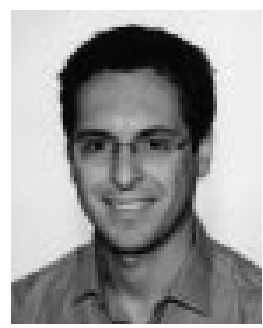

Giuseppe C. Calafiore was born in Torino, Italy in December 1969. He received the "Laurea" degree in electrical engineering from Politecnico di Torino in 1993, and the Doctorate degree in information and system theory from Politecnico di Torino, in 1997. Since 1998, he holds the position of an Assistant Professor at Dipartimento di Automatica e Informatica, Politecnico di Torino.

Dr. Calafiore held visiting positions at Information Systems Laboratory, Standford University, in 1995, at Ecole Nationale Supéerieure de Techniques Avanceées (ENSTA), Paris, in 1998, and at the University of California at Berkeley, in 1999. His research interests are in the fields of convex optimization, randomized algorithms, and analysis, identification, prediction and control of uncertain systems, with applications ranging from finance and economic systems, to robust control, pattern recognition and robotics.

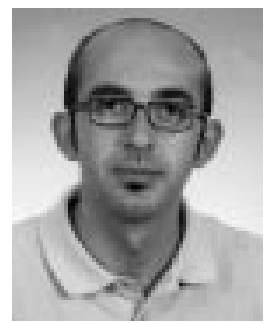

Fabrizio Dabbene received the Laurea degree in electronic engineering in 1995 and the Ph.D. degree in systems and computer engineering in 1999, both from Politecnico di Torino. In 1997, he has been a Visiting Researcher at the Department of Electrical Engineering, University of Iowa. He is currently holding a postdoctoral position at Politecnico di Torino, where he teaches several courses in Systems and Control. Dr. Dabbene also holds a research collaboration with the research center IRITI of the National Research Council (CNR) of Italy. His research interests include robust control and identification of uncertain systems, randomized algorithms and optimization. 


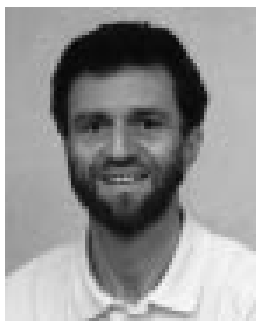

Roberto Tempo (M'90-SM'98-F'00) was born in Cuorgné, Italy, in 1956. In 1980 he graduated in electrical engineering at Politecnico di Torino, Italy. From 1981 to 1983 he was with the Dipartimento di Automatica e Informatica, Politecnico di Torino. In 1984 he joined the National Research Council of Italy (CNR) at the research institute IRITI, Torino, where he is a Director of Research of Systems and Computer Engineering since 1991 and an elected member of the Scientific Council. He has held visiting and research positions at Columbia University in New York, German Aerospace Research Organization in Oberpfaffenhofen and University of Wisconsin at Madison.

Dr. Tempo's research activities are mainly focused on robustness analysis and control of uncertain systems and identification of complex systems subject to bounded errors. In 1993, he received the "Outstanding Paper Prize Award" from the International Federation of Automatic Control (IFAC) for a paper published in Automatica.

Dr. Tempo has been an Associate Editor of Systems and Control Letters and he is currently an Editor of Automatica and an Associate Editor of IEEE TRANSACtions on AutOMatic CONTROL. He has served as program Vice-Chair for short papers of the IEEE Conference on Decision and Control, 1999. He is a Fellow of the IEEE and a member of the EUCA (European Union of Control Associations) Council. 